\title{
Cuspidal divisor class groups of non-split Cartan modular curves
}

\author{
Pierfrancesco Carlucci \\ Dipartimento di Matematica \\ Universitá degli Studi di Roma Tor Vergata \\ Via della Ricerca Scientifica 1, 00133, Rome, Italy \\ E-mail: pieffecar@libero.it
}

30 April, 2016

\begin{abstract}
I find an explicit description of modular units in terms of Siegel functions for the modular curves $X_{n s}^{+}\left(p^{k}\right)$ associated to the normalizer of a non-split Cartan subgroup of level $p^{k}$ where $p \neq 2,3$ is a prime. The Cuspidal Divisor Class Group $\mathfrak{C}_{n s}^{+}\left(p^{k}\right)$ on $X_{n s}^{+}\left(p^{k}\right)$ is explicitly described as a module over the group ring $R=\mathbb{Z}\left[\left(\mathbb{Z} / p^{k} \mathbb{Z}\right)^{*} /\{ \pm 1\}\right]$. In this paper I give a formula involving generalized Bernoulli numbers $B_{2, \chi}$ for $\left|\mathfrak{C}_{n s}^{+}\left(p^{k}\right)\right|$.
\end{abstract}

\section{Motivation and overview}

Let $X_{n s}^{+}(n)$ be the modular curve associated to the normalizer of a nonsplit Cartan subgroup of level $n$. One noteworthy reason for studying these curves is the Serre's uniformity problem over $\mathbb{Q}$ stating that there exists a constant $C>0$ so that, if $E$ is an elliptic curve over $\mathbb{Q}$ without complex multiplication, then the Galois representation:

$$
\rho_{E, p}: \operatorname{Gal}(\overline{\mathbb{Q}}, \mathbb{Q}) \rightarrow \mathrm{GL}_{2}\left(\mathbb{F}_{p}\right)
$$

attached to the elliptic curve $E$ is onto for all primes $p>C$ (see [16] and [7, pag. 198]). If the Galois representation were not surjective, its image would

2010 Mathematics Subject Classification: Primary 11G16; Secondary 11B68, $13 \mathrm{C} 20$.

Key words and phrases: Siegel Functions, Modular Units, Cuspidal Divisor Class Group, Non-Split Cartan Curves, Generalized Bernoulli Numbers. 
be contained in one of the maximal proper subgroups of $\mathrm{GL}_{2}\left(\mathbb{F}_{p}\right)$. These subgroups are:

1. A Borel subgroup;

2. The normalizer of a split Cartan subgroup;

3. The normalizer of a non-split Cartan subgroup;

4. A finite list of exceptional subgroups.

Serre himself showed that if $p>13$ the image of $\rho_{E, p}$ is not contained in an exceptional subgroup. Mazur in [12] and Bilu-Parent-Rebolledo in [2] presented analogous results for Borel subgroups and split Cartan subgroups respectively. The elliptic curves over $\mathbb{Q}$ for which the image of the Galois representation is contained in the normalizer of a non-split Cartan subgroup are parametrized by the non-cuspidal rational points of $X_{n s}^{+}(p)$. Thus the open case of Serre's uniformity problem can be reworded in terms of determining whether there exist $\mathbb{Q}$-rational points on $X_{n s}^{+}(p)$, that do not arise from elliptic curves with complex multiplication.

This paper focuses on an aspect of the curves $X_{n s}^{+}\left(p^{k}\right)$ that has never been treated before: their Cuspidal Divisor Class Group $\mathfrak{C}_{n s}^{+}\left(p^{k}\right)$, a finite subgroup of the Jacobian $J_{n s}^{+}\left(p^{k}\right)$ whose support is contained in the set of cusps of $X_{n s}^{+}\left(p^{k}\right)$. Let $\mathfrak{D}_{n s}^{+}\left(p^{k}\right)$ be the free abelian group generated by the cusps of $X_{n s}^{+}\left(p^{k}\right)$, let $\mathfrak{D}_{n s}^{+}\left(p^{k}\right)_{0}$ be its subgroup consisting of elements of degree 0 and let $\mathfrak{F}_{n s}^{+}\left(p^{k}\right)$ be the group of divisors of modular units of $X_{n s}^{+}\left(p^{k}\right)$, i.e. those modular functions on $X_{n s}^{+}\left(p^{k}\right)$ in the modular function field $F_{p^{k}}$, which have no zeros and poles in the upper-half plane. We define:

$$
\mathfrak{C}_{n s}^{+}\left(p^{k}\right):=\mathfrak{D}_{n s}^{+}\left(p^{k}\right)_{0} / \mathfrak{F}_{n s}^{+}\left(p^{k}\right) .
$$

In [8] Kubert and Lang gave an explicit and complete description of the group of modular units of $X\left(p^{k}\right)$ in terms of Siegel functions $g_{a}(\tau)$ (see [9] or [18]) with $a \in \frac{1}{p^{k}} \mathbb{Z}^{2} \backslash \mathbb{Z}^{2}$. We will define the set of functions

$$
\left\{G_{h}^{+}(\tau)\right\}_{h \in\left(\left(\mathbb{Z} / p^{k} \mathbb{Z}\right)^{*} /\{ \pm 1\}\right)}
$$

in terms of classical Siegel functions and we will prove the following result:

Theorem 6.5 If $p \neq 2,3$, the group of modular units of the modular curve $X_{n s}^{+}\left(p^{k}\right)$ consists (modulo constants) of power products:

$$
g(\tau)=\prod_{h \in\left(\left(\mathbb{Z} / p^{k} \mathbb{Z}\right)^{*} /\{ \pm 1\}\right)} G_{h}^{+n_{h}^{+}}(\tau)
$$


where

$$
G_{h}^{+}(\tau)=\prod_{t \in\left(\left(\mathbf{O}_{K} / p^{k} \mathbf{O}_{K}\right)^{*} /\{ \pm 1\}\right), \pm|t|=h} g_{[t]}(\tau)
$$

and $d=\frac{12}{\operatorname{gcd}(12, p+1)}$ divides $\sum_{h} n_{h}^{+}$.

In [7, Chapter 5] Kubert and Lang studied the Cuspidal Divisor Class Group on the modular curve $X\left(p^{k}\right)$. Since their description utilizes the parametrization of the set of cusps of $X\left(p^{k}\right)$ by the elements of the quotient $C_{n s}\left(p^{k}\right) /\{ \pm 1\}$, it appears natural to develop and extend their techniques to non-split Cartan modular curves. Kubert and Lang proved the following:

Theorem 4.6 If $p \geq 5$ consider $R:=\mathbb{Z}\left[C_{n s}\left(p^{k}\right) /\{ \pm 1\}\right]$ and let $R_{0}$ be the ideal of $R$ consisting of elements of degree 0. The Cuspidal Divisor Class Group $\mathfrak{C}_{p^{k}}$ on $X\left(p^{k}\right)$ is an $R$-module, more precisely there exists a Stickelberger element $\theta \in \mathbb{Q}\left[C_{n s}\left(p^{k}\right) /\{ \pm 1\}\right]$ such that, under the identification of the group $C_{n s}\left(p^{k}\right) /\{ \pm 1\}$ with the set of cusps at level $p^{k}$, the ideal $R \cap R \theta$ corresponds to the group of divisors of units in the modular function field $F_{p^{k}}$ and:

$$
\mathfrak{C}_{p^{k}} \cong R_{0} / R \cap R \theta
$$

In this theorem the authors exhibited an isomorphism reminding to a classical result in cyclotomic fields theory. Let $J$ be a fractional ideal of $\mathbb{Q}\left(\zeta_{m}\right)$ and $G=\operatorname{Gal}\left(\mathbb{Q}\left(\zeta_{m}\right) / \mathbb{Q}\right) \cong(\mathbb{Z} / m \mathbb{Z})^{*}$. Consider $\mathbb{Z}[G]$ acting on the ideals and ideal classes in the natural way: if $x=\sum_{\sigma} x_{\sigma} \sigma$ then $J^{x}:=\prod_{\sigma}\left(J^{\sigma}\right)^{x_{\sigma}}$. We have the following result:

Stickelberger's Theorem [20, pag. 333] Define the Stickelberger element:

$$
\theta=\sum_{a \bmod m,(a, m)=1}\left\langle\frac{a}{m}\right\rangle \sigma_{a}^{-1} \in \mathbb{Q}[G] .
$$

The Stickelberger ideal $\mathbb{Z}[G] \cap \theta \mathbb{Z}[G]$ annihilates the ideal class group of $\mathbb{Q}\left(\zeta_{m}\right)$.

Along these lines, the main result can be summarized as follows:

Main Theorem 7.1 Consider $p \geq 5, H:=\left(\mathbb{Z} / p^{k} \mathbb{Z}\right)^{*} /\{ \pm 1\}$ and $w$ a generator of $H$. There exists a Stickelberger element

$$
\theta:=\frac{p^{k}}{2} \sum_{i=1}^{\frac{p-1}{2} p^{k-1}} \sum_{ \pm|s|=w^{i}, s \in\left(\left(\mathbf{O}_{K} / p^{k} \mathbf{O}_{K}\right)^{*} /\{ \pm 1\}\right.} B_{2}\left(\left\langle\frac{\frac{1}{2}(s+\bar{s})}{p^{k}}\right\rangle\right) w^{-i} \in \mathbb{Q}[H]
$$


such that, under the identification of the group $H$ with the set of cusps of $X_{n s}^{+}\left(p^{k}\right)$, the ideal $\mathbb{Z}[H] \theta \cap \mathbb{Z}[H]$ represents the group of divisors of units of $X_{n s}^{+}\left(p^{k}\right)$. The Cuspidal Divisor Class Group on $X_{n s}^{+}\left(p^{k}\right)$ is a module over $\mathbb{Z}[H]$ and, more precisely, we have:

$$
\mathfrak{C}_{n s}^{+}\left(p^{k}\right) \cong \mathbb{Z}_{0}[H] /(\mathbb{Z}[H] \theta \cap \mathbb{Z}[H])
$$

From the previous statement we will show another result which has a counterpart in cyclotomic field theory.

Theorem 7.4 For any character $\chi$ of $C_{n s}\left(p^{k}\right) /\{ \pm I\} \quad$ (identified with an even character of $\left.C_{n s}\left(p^{k}\right)\right)$, we let:

$$
B_{2, \chi}=\sum_{\alpha \in C_{n s}\left(p^{k}\right) /\{ \pm I\}} B_{2}\left(\left\langle\frac{T(\alpha)}{p^{k}}\right\rangle\right) \chi(\alpha)
$$

where $B_{2}(t)=t^{2}-t+\frac{1}{6}$ is the second Bernoulli polynomial and $T$ is a certain $\left(\mathbb{Z} / p^{k} \mathbb{Z}\right)$-linear map. Then we have:

$$
\left|\mathfrak{C}_{n s}^{+}\left(p^{k}\right)\right|=24 \frac{\prod \frac{p^{k}}{2} B_{2, \chi}}{\operatorname{gcd}(12, p+1)(p-1) p^{k-1}}
$$

where the product runs over all nontrivial characters $\chi$ of $C_{n s}\left(p^{k}\right) / \pm I$ such that $\chi(M)=1$ for every $M \in C_{n s}\left(p^{k}\right)$ with $\operatorname{det} M= \pm 1$.

In particular, for $k=1$ let $\omega$ be a generator of the character group of $C_{n s}(p)$ and $v$ a generator of $\mathbb{F}_{p^{2}}^{*}$. Then:

$$
\begin{gathered}
\left|\mathfrak{C}_{n s}^{+}(p)\right|=\frac{24}{(p-1) \operatorname{gcd}(12, p+1)} \prod_{j=1}^{\frac{p-3}{2}} \frac{p}{2} B_{2, \omega(2 p+2) j}= \\
=\frac{576\left|\operatorname{det}\left[\frac{p}{2}\left(\sum_{l=0}^{p} B_{2}\left(\left\langle\frac{\frac{1}{2} \operatorname{Tr}\left(v^{i-j+l \frac{p-1}{2}}\right)}{p}\right\rangle\right)-\frac{p+1}{6}\right)\right]_{1 \leq i, j \leq \frac{p-1}{2}}\right|}{(p-1)^{2} p(p+1) \operatorname{gcd}(12, p+1)} .
\end{gathered}
$$

This theorem could be considered analogous to the relative class number formula [20, Theorem 4.17]:

$$
h_{m}^{-}=Q w \prod_{\chi \text { odd }}-\frac{1}{2} B_{1, \chi}
$$


where $Q=1$ if $m$ is a prime power and $Q=2$ otherwise, $w$ is the number of roots of unity in $\mathbb{Q}\left(\zeta_{m}\right)$ and we encounter the classical generalized Bernoulli numbers:

$$
B_{1, \chi}:=\sum_{a=1}^{m} \chi(a) B_{1}\left(\frac{a}{m}\right)=\frac{1}{m} \sum_{a=1}^{m} \chi(a) a \text { for } \chi \neq 1 .
$$

In the last section we will explicitly calculate $\left|\mathfrak{C}_{n s}^{+}(p)\right|$ for some $p \leq 101$. Consider the isogeny (cfr. [4, Paragraph 6.6]):

$$
J_{0}^{+ \text {new }}\left(p^{2}\right) \longrightarrow \bigoplus_{f} A_{p, f}^{\prime}
$$

where the sum is taken over the equivalence classes of newforms $f \in S_{2}\left(\Gamma_{0}^{+}\left(p^{2}\right)\right)$. From Theorems 8.3, 8.4 and 8.5 we deduce that:

$$
\left|\mathfrak{C}_{n s}^{+}(p)\right| \text { divides } \prod_{f} \underset{\substack{q \operatorname{prime}, q \nmid\left|\mathfrak{C}_{n s}^{+}(p)\right|, q \equiv \pm 1 \bmod p}}{\operatorname{gcd}}\left|A_{p, f}^{\prime}\left(\mathbb{F}_{q}\right)\right| .
$$

Using the modular form database of W.Stein, we will find out that for $p \leq 31$ :

$$
\left|\mathfrak{C}_{n s}^{+}(p)\right|=\prod_{f} \underset{\substack{q<500 \text { prime, } \\ q \equiv \pm 1 \bmod p}}{\operatorname{gcd}}\left|A_{p, f}^{\prime}\left(\mathbb{F}_{q}\right)\right| .
$$

\section{Galois groups of modular function fields}

Following [19, Chapter 1], let $\mathbb{H}=\{x+i y \mid y>0 ; x, y \in \mathbb{R}\}$ be the upperhalf plane and $n$ a positive integer. The principal congruence subgroup of level $n$ is the subgroup of $S L_{2}(\mathbb{Z})$ defined as follows:

$$
\Gamma(n):=\left\{\left(\begin{array}{ll}
a & b \\
c & d
\end{array}\right) \in S L_{2}(\mathbb{Z}): a \equiv d \equiv 1, b \equiv c \equiv 0 \bmod n\right\} .
$$

Then the quotient space $\Gamma(n) \backslash \mathbb{H}$ is complex analytically isomorphic to an affine curve $Y(n)$ that can be compactified by considering $\mathbb{H}^{*}:=\mathbb{H} \cup \mathbb{Q} \cup\{\infty\}$ and by taking the extended quotient:

$$
X(n)=\Gamma(n) \backslash \mathbb{H}^{*}=Y(n) \cup \Gamma(n) \backslash(\mathbb{Q} \cup\{\infty\}) .
$$

The points $\Gamma(n) \tau$ in $\Gamma(n) \backslash(\mathbb{Q} \cup\{\infty\})$ are called the cusps of $\Gamma(n)$ and can be described by the fractions $s=\frac{a}{c}$ with $0 \leq a \leq n-1,0 \leq c \leq n-1$ and $\operatorname{gcd}(a, c)=1$. As a consequence, it is not difficult to infer that $X(n)$ has $\frac{1}{2} n^{2} \prod_{p \mid n}\left(1-\frac{1}{p^{2}}\right)$ cusps. 
Let $F_{n, \mathbb{C}}$ the field of modular functions of level $n$. A classical result states that $F_{1, \mathbb{C}}=\mathbb{C}(j)$ where $j$ is the Klein's $j$-invariant. We shall now find generators for $F_{n, \mathbb{C}}$. Consider:

$$
f_{0}(w ; \tau)=-2^{7} 3^{5} \frac{g_{2}(\tau) g_{3}(\tau)}{\Delta(\tau)} \wp(w ; \tau, 1),
$$

where $\Delta$ is the modular discriminant, $\wp$ is the Weierstrass elliptic function, $\tau \in \mathbb{H}, w \in \mathbb{C}$ and $g_{2}=60 G_{4}$ and $g_{3}=140 G_{6}$ are constant multiples of the Eisenstein series:

$$
G_{2 k}(\tau)=\sum_{(m, n) \in \mathbf{Z}^{2} \backslash(0,0)} \frac{1}{(m+n \tau)^{2 k}}
$$

For $r, s \in \mathbb{Z}$ and not both divisible by $n$ we define $f_{r, s}=f_{0}\left(\frac{r \tau+s}{n} ; \tau\right)$. Whereas the Weierestrass $\wp$-function is elliptic with respect to the lattice $[\tau, 1]$ it follows that $f_{r, s}$ depends only on the residue of $r, s \bmod n$. Thus, it is convenient to use a notation emphasizing this property. If $a=\left(a_{1}, a_{2}\right) \in \mathbb{Q}^{2}$ but $a \notin \mathbb{Z}^{2}$ we call the functions $f_{a}(\tau)=f_{0}\left(a_{1} \tau+a_{2} ; \tau\right)$ the Fricke functions. They depend only on the residue class of $a \bmod \mathbb{Z}^{2}$.

Theorem 2.1. We have:

$$
\operatorname{Gal}\left(F_{n, \mathbb{C}}, F_{1, \mathbb{C}}\right) \cong S L_{2}(\mathbb{Z} / n \mathbb{Z}) /\{ \pm I\}
$$

Proof. There is a surjective homeomorphism (see [4, pag.279] and [9, pag.65]):

$$
\begin{gathered}
\theta: S L_{2}(\mathbb{Z}) \longrightarrow \operatorname{Aut}(\mathbb{C}(X(n))), \\
\gamma \longmapsto\left(f \longmapsto f^{(\theta(\gamma))}=f \circ \gamma\right) .
\end{gathered}
$$

From $\operatorname{Ker}(\theta)= \pm \Gamma(n)$ and the relations $f_{a}(\gamma(\tau))=f_{a \gamma}(\tau)$ it follows easily that $\operatorname{Gal}\left(F_{n, \mathbb{C}}, F_{1, \mathbb{C}}\right) \cong \Gamma(1) / \pm \Gamma(n) \cong S L_{2}(\mathbb{Z} / n \mathbb{Z}) /\{ \pm I\}$.

We say that a modular form in $F_{n, \mathbb{C}}$ is defined over a field if all the coefficients of its $q$-expantion lie in that field and analogously for every $\operatorname{Gal}\left(F_{n, \mathbb{C}}, F_{1, \mathbb{C}}\right)$-conjugate of the form. Let:

$F_{n}=$ function field on $X(n)$ consisting of those functions which are defined over the $n$-th cyclotomic field $\mathbb{Q}_{n}=\mathbb{Q}\left(\zeta_{n}\right)$.

Theorem 2.2. The field $F_{n}$ has the following properties:

(1) $F_{n}$ is a Galois extension of $F_{1}=\mathbb{Q}(j)$.

(2) $F_{n}=\mathbb{Q}\left(j, f_{r, s}\right)_{\text {all }(r, s) \in \frac{1}{n} \mathbb{Z}^{2} \backslash \mathbb{Z}^{2}}$.

(3) For every $\gamma \in G L_{2}(\mathbb{Z} / n \mathbb{Z})$ the map $f_{a} \mapsto f_{a \gamma}$ gives an element of 
$\operatorname{Gal}\left(F_{n}, \mathbb{Q}(j)\right)$ which we write $\theta(\gamma)$. Then $\gamma \mapsto \theta(\gamma)$ induces an isomorphism of $G L_{2}(\mathbb{Z} / n \mathbb{Z}) / \pm I$ to $G a l\left(F_{n}, \mathbb{Q}(j)\right)$. The subgroup $S L_{2}(\mathbb{Z} / n \mathbb{Z}) / \pm I$ operates on a modular function by composition with the natural action of $S L_{2}(\mathbb{Z})$ on the upper half-plane $\mathbb{H}$.

Furthermore the group of matrices $\left(\begin{array}{ll}1 & 0 \\ 0 & d\end{array}\right)$ operates on $F_{n}$ as follows: for $d \in(\mathbb{Z} / n \mathbb{Z})^{*}$ consider the automorphism $\sigma_{d}$ of $\mathbb{Q}_{n}$ such that $\sigma_{d}\left(\zeta_{n}\right)=\zeta_{n}^{d}$. Then $\sigma_{d}$ extends to $F_{n}$ by operating on the coefficients of the power series expansions:

$$
\sigma_{d}\left(\sum a_{i} q^{i / n}\right)=\sum \sigma_{d}\left(a_{i}\right) q^{i / n} \text { with } q=e^{2 \pi i \tau} .
$$

If $(r, s) \in \frac{1}{n} \mathbb{Z}^{2} \backslash \mathbb{Z}^{2}$, we have: $\sigma_{d}\left(f_{r, s}(\tau)\right)=f_{r, s d}(\tau)$.

Proof. [17, Theorem 6.6]

\section{Modular Units and Manin-Drinfeld Theo- rem}

In this paper we will focus our attention on the modular units of $X(n)$. In other words, the invertible elements of the integral closure of $\mathbb{Q}[j]$ in $F_{n}$. The only pole of $j(\tau)$ is at infinity. So, from the algebraic characterization of the integral closure as the intersection of all valuation subrings containing the given ring, the modular units in $F_{n}$ are exactly the modular functions which have poles and zeros exclusively at the cusps of $X(n)$.

Let $\mathfrak{D}_{n} \simeq \bigoplus_{\text {cusps }} \mathbb{Z}$ be the free abelian group of rank $\frac{1}{2} n^{2} \prod_{p \mid n}\left(1-\frac{1}{p^{2}}\right)$ generated by the cusps of $X(n)$. Let $\mathfrak{D}_{n, 0}$ be its subgroup consisting of elements of degree 0 and let $\mathfrak{F}_{n}$ be the subgroup generated by the divisors of modular units in the modular function field $F_{n}$. The quotient group:

$$
\mathfrak{C}_{n}:=\mathfrak{D}_{n, 0} / \mathfrak{F}_{n}
$$

is called the Cuspidal Divisor Class Group on $X(n)$. The previous definition generalizes mutatis mutandis to every modular curve $X_{\Gamma}$ where $\Gamma$ is a modular subgroup. Manin and Drinfeld proved that:

Theorem 3.1. If $\Gamma$ is a congruence subgroup then all divisors of degree 0 whose support is a subset of the set of cusps of $X_{\Gamma}$ have a multiple that is a principal divisor. In other word if $x_{1}, x_{2} \in X_{\Gamma}$ are cusps, then $x_{1}-x_{2}$ has finite order in the jacobian variety $\operatorname{Jac}\left(X_{\Gamma}\right)$. 
Proof. Let $x_{1}, x_{2}$ two cusps in $X_{\Gamma}$. Denote by $\left\{x_{1}, x_{2}\right\} \in\left(\Omega^{1}\left(X_{\Gamma}\right)\right)^{*}$ the functional on the space of differential of the first kind given by:

$$
\left\{x_{1}, x_{2}\right\}: \omega \mapsto \int_{x_{1}}^{x_{2}} \omega .
$$

A priori we have $\left\{x_{1}, x_{2}\right\} \in H_{1}\left(X_{\Gamma}, \mathbb{R}\right)$. Manin and Drinfeld showed that it lies in $H_{1}\left(X_{\Gamma}, \mathbb{Q}\right)$. Cf. [5], [10, Chapter IV] and [11].

\section{Siegel Functions and Cuspidal Divisor Class Groups}

Let $n=p^{k}$ with $p \geq 5$ prime. Following [7] we will give an explicit description of modular units of $X(n)$ and its cuspidal divisor class group.

Let $L$ a lattice in $\mathbb{C}$. Define the Weierstrass sigma function:

$$
\sigma_{L}(z)=z \prod_{\substack{\omega \in L \\ \omega \neq 0}}\left(1-\frac{z}{\omega}\right) e^{z / \omega+\frac{1}{2}(z / \omega)^{2}},
$$

which has simple zeros at all non-zero lattice points. Define:

$$
\begin{gathered}
\zeta_{L}(z)=\frac{d}{d z} \log \left(\sigma_{L}(z)\right)=\frac{1}{z}+\sum_{\substack{\omega \in L \\
\omega \neq 0}}\left[\frac{1}{z-\omega}+\frac{1}{\omega}+\frac{z}{\omega^{2}}\right], \\
\wp_{L}(z)=-\zeta_{L}^{\prime}(z)=\frac{1}{z^{2}}+\sum_{\substack{\omega \in L \\
\omega \neq 0}}\left[\frac{1}{(z-\omega)^{2}}-\frac{1}{\omega^{2}}\right] .
\end{gathered}
$$

If $\omega \in L$, by virtue of the periodicity of $\wp_{L}$ we obtain $\frac{d}{d z} \zeta_{L}(z+\omega)=\frac{d}{d z} \zeta_{L}(z)$, whence follows the existence of a $\mathbb{R}$-linear function $\eta_{L}(z)$ such that:

$$
\zeta_{L}(z+\omega)=\zeta_{L}(z)+\eta_{L}(\omega)
$$

For $L=[\tau, 1]$ (with $\tau \in \mathbb{H}$ ) and $a=\left(a_{1}, a_{2}\right) \in \mathbb{Q}^{2} \backslash \mathbb{Z}^{2}$ we define the Klein forms:

$$
\mathfrak{k}_{a}(\tau)=e^{-\eta_{L}\left(a_{1} \tau+a_{2}\right)} \sigma_{L}\left(a_{1} \tau+a_{2}\right) .
$$

Note that $z=a_{1} \tau+a_{2} \notin L=[\tau, 1]$ so we know directly from their definition that the Klein forms are holomorphic functions which have no zeros and poles on the upper half plane.

When $\Gamma$ is a congruence subgroup and $k$ is an integer, we will say that a holomorphic function $f(\tau)$ on $\mathbb{H}$ is a nearly holomorphic modular form for 
$\Gamma$ of weight $k$ if:

(i) $f(\gamma(\tau))=(r \tau+s)^{k} f(\tau)$ for all $\gamma=\left(\begin{array}{ll}p & q \\ r & s\end{array}\right) \in \Gamma$;

(ii) $f(\tau)$ is meromorphic at every cusp.

Proposition 4.1. Let $a=\left(a_{1}, a_{2}\right) \in \mathbb{Q}^{2} \backslash \mathbb{Z}^{2}$ and $b=\left(b_{1}, b_{2}\right) \in \mathbb{Z}^{2}$. The Klein Forms $\mathfrak{k}_{a}(\tau)$ have the following properties:

(1) $\mathfrak{k}_{-a}(\tau)=-\mathfrak{k}_{a}(\tau)$;

(2) $\mathfrak{k}_{a+b}=\epsilon(a, b) \mathfrak{k}_{a}(\tau)$ with $\epsilon(a, b)=(-1)^{b_{1} b_{2}+b_{1}+b_{2}} e^{-\pi i\left(b_{1} a_{2}-b_{2} a_{1}\right)}$;

(3) For every $\gamma=\left(\begin{array}{ll}p & q \\ r & s\end{array}\right) \in S L_{2}(\mathbb{Z})$ we have:

$$
\mathfrak{k}_{a}(\gamma(\tau))=\mathfrak{k}_{a}\left(\frac{p \tau+q}{r \tau+s}\right)=\frac{\mathfrak{k}_{a \gamma}(\tau)}{r \tau+s}=\frac{\mathfrak{k}_{\left(a_{1} p+a_{2} r, a_{1} q+a_{2} s\right)}(\tau)}{r \tau+s}
$$

(4) If $n \geq 2$ and $a \in \frac{1}{n} \mathbb{Z}^{2} \backslash \mathbb{Z}^{2}$ then $\mathfrak{k}_{a}(\tau)$ is a nearly holomorphic modular form for $\Gamma\left(2 n^{2}\right)$ of weight -1 .

(5) Let $n \geq 3$ odd and $\left\{m_{a}\right\}_{a \in \frac{1}{n} \mathbb{Z}^{2} \backslash \mathbb{Z}^{2}}$ a family of integers such that $m_{a} \neq 0$ occurs only for finitely many a. Then the product of Klein form:

$$
\prod_{a \in \frac{1}{n} \mathbb{Z}^{2} \backslash \mathbb{Z}^{2}} \mathfrak{k}_{a}^{m_{a}}(\tau)
$$

is a nearly holomorphic modular form for $\Gamma(n)$ of weight $-\sum_{a} m_{a}$ if and only if:

$$
\sum_{a} m_{a}\left(n a_{1}\right)^{2} \equiv \sum_{a} m_{a}\left(n a_{2}\right)^{2} \equiv \sum_{a} m_{a}\left(n a_{1}\right)\left(n a_{2}\right) \equiv 0 \quad \bmod n
$$

Proof. Property (2) is nothing more than a reformulation of the Legendre relation: $\eta_{[\tau, 1]}(1) \tau-\eta_{[\tau, 1]}(\tau)=2 \pi i$. Property (5) is discussed in [7, Chapter 3, Paragraph 4].

For more details see: [7, Chapters 2 and 3] or [9, Chapter 19].

We are now ready to define the Siegel function:

$$
g_{a}(\tau)=\mathfrak{k}_{a}(\tau) \Delta(\tau)^{1 / 12},
$$

where $\Delta(\tau)$ is the square of the Dedekind eta funtion $\eta(\tau)$ (not to be mistaken for the aforementioned $\left.\eta_{L}(\tau)\right)$ :

$$
\eta(\tau)^{2}=2 \pi i q^{1 / 12} \prod_{k=1}^{\infty}\left(1-q^{n}\right)^{2} \text { with } q=e^{2 \pi i \tau}
$$


Proposition 4.2. The set of functions $\left\{h_{a}(\tau)=g_{a}(\tau)^{12 n}\right\}_{a \in \frac{1}{n} \mathbb{Z}^{2} \backslash \mathbb{Z}^{2}}$ constitute a Fricke family. Just like the Fricke functions $f_{a}(\tau)$ of Theorem 2.2 we have: $h_{a}(\tau) \in F_{n}$, for every $\gamma \in S L_{2}(\mathbb{Z})$ we have $h_{a}(\gamma(\tau))=h_{a \gamma}(\tau)$ and in addition if $\sigma_{d} \in \operatorname{Gal}\left(\mathbb{Q}_{n}, \mathbb{Q}\right)$ then $\sigma_{d}\left(h_{a_{1}, a_{2}}(\tau)\right)=h_{a_{1}, d a_{2}}(\tau)$. In other words, the Siegel functions, raised to the appropriate power, are permuted by the elements of the Galois Group $\operatorname{Gal}\left(F_{n}, \mathbb{Q}(j)\right)$.

Proof. [7, Chapter 2] or [18].

Theorem 4.3. Assume that $n=p^{k}$ for $p \neq 2,3$. Then the units in $F_{n}$ (modulo constants) consist of the power products:

$$
\prod_{a \in \frac{1}{n} \mathbb{Z}^{2} \backslash \mathbb{Z}^{2}} g_{a}^{m_{a}}(\tau)
$$

with:

$$
\sum_{a} m_{a}\left(n a_{1}\right)^{2} \equiv \sum_{a} m_{a}\left(n a_{2}\right)^{2} \equiv \sum_{a} m_{a}\left(n a_{1}\right)\left(n a_{2}\right) \equiv 0 \quad \bmod n
$$

and

$$
\sum_{a} m_{a} \equiv 0 \quad \bmod 12
$$

In addition, if $k \geq 2$ it is not restrictive to consider power products of Siegel functions $g_{a}$ with primitive index $a=\left(a_{1}, a_{2}\right)$, namely such that $p^{k-1} a \notin \mathbb{Z}^{2}$.

Proof. See [8], 7, Theorem 3.2, Chapter 2], [7, Theorem 5.2, Chapter 3] and [7, Theorem 1.1, Chapter 4]. The last assertion is a consequence of the distribution relations discussed in [7, pp. 17-23].

Following [7] it will be useful to decompose $\operatorname{Gal}\left(F_{p^{k}}, \mathbb{Q}(j)\right)$. Let $\mathfrak{o}_{p}$ the ring of integers in the unramified quadratic extension of the $p$-adic field $\mathbb{Q}_{p}$. The group of units $\mathfrak{o}_{p}^{*}$ acts on $\mathfrak{o}_{p}$ by multiplication and after choosing a basis of $\mathfrak{o}_{p}$ over the $p$-adic ring $\mathbb{Z}_{p}$, we obtain an embedding:

$$
\mathfrak{o}_{p}^{*} \longrightarrow G L_{2}\left(\mathbb{Z}_{p}\right) .
$$

We call the image in $G L_{2}\left(\mathbb{Z}_{p}\right)$ the Cartan Group at the prime $p$ and indicate it by $C_{p}$. It is worth noting that the elements of $\mathfrak{o}_{p}^{*}$, written in terms of a basis of $\mathfrak{o}_{p}$ over $\mathbb{Z}_{p}$, are characterized by the fact that at least one of the two coefficients is a unit.

Consider now $G L_{2}\left(\mathbb{Z}_{p}\right)$ as operating on $\mathbb{Z}_{p}^{2}$ on the left and denote by $G_{p, \infty}$ the isotropy group of $\left(\begin{array}{l}1 \\ 0\end{array}\right)$. Obviously we have: 


$$
G_{p, \infty}=\left\{\left(\begin{array}{ll}
1 & b \\
0 & d
\end{array}\right) b \in \mathbb{Z}_{p}, d \in \mathbb{Z}_{p}^{*}\right\}
$$

Since $C_{p}$ operates simply transitively on the set of primitive elements (that is: vectors whose coordinates are not both divisibile by $p$ ) we have the following decomposition:

$$
G L_{2}\left(\mathbb{Z}_{p}\right) /\{ \pm I\}=\left(C_{p} /\{ \pm I\}\right) G_{p, \infty}
$$

For each integer $k$ we define the reduction of the Cartan Group $C_{p} \bmod p^{k}$ :

$$
C\left(p^{k}\right)=C_{p} / p^{k} C_{p}
$$

and let $G_{\infty}\left(p^{k}\right)$ the reduction of $G_{p, \infty} \bmod p^{k}$ :

$$
G_{\infty}\left(p^{k}\right)=\left\{\left(\begin{array}{ll}
1 & b \\
0 & d
\end{array}\right) b \in \mathbb{Z} / p^{k} \mathbb{Z}, d \in\left(\mathbb{Z} / p^{k} \mathbb{Z}\right)^{*}\right\} .
$$

We can now reformulate the previous decomposition as follows:

$$
\operatorname{Gal}\left(F_{p^{k}}, \mathbb{Q}(j)\right) \simeq G L_{2}\left(\mathbb{Z} / p^{k} \mathbb{Z}\right) /\{ \pm I\}=\left(C\left(p^{k}\right) /\{ \pm I\}\right) G_{\infty}\left(p^{k}\right)
$$

The embedding:

$$
F_{p^{k}} \hookrightarrow \mathbb{Q}\left(\zeta_{p^{k}}\right)\left(\left(q^{1 / p^{k}}\right)\right)
$$

enables us to mesaure for each modular function $f(\tau) \in F_{p^{k}}$ its order at $\Gamma\left(p^{k}\right) \infty$ in term of the local parameter $q^{1 / p^{k}}$.

Proposition 4.4. If $a \in \frac{1}{p^{k}} \mathbb{Z}^{2} \backslash \mathbb{Z}^{2}$, the q-expansion of the Siegel functions shows that:

$$
\operatorname{ord}_{\infty}\left(g_{a}(\tau)\right)^{12 p^{k}}=6 p^{2 k} B_{2}\left(\left\langle a_{1}\right\rangle\right)
$$

where $B_{2}(X)=X^{2}-X+\frac{1}{6}$ is the second Bernoulli polynomial and $\langle X\rangle$ is the fractional part of $X$.

Proof. [9, Chapter 19].

For every automorphism $\sigma \in \operatorname{Gal}\left(F_{p^{k}}, \mathbb{Q}(j)\right)$ and each $h(\tau) \in F_{p^{k}}$ we have the prime $\sigma^{-1}(\infty)$ which is such that:

$$
\operatorname{ord}_{\sigma^{-1}(\infty)}(h(\tau))=\operatorname{ord}_{\infty} \sigma(h(\tau))
$$

and if $\sigma \in G_{\infty}\left(p^{k}\right)$ :

$$
\operatorname{ord}_{\infty}(h(\tau))=\operatorname{ord}_{\infty} \sigma(h(\tau))
$$


so we may identify the cusps of $X\left(p^{k}\right)$ with the elements of the Cartan Group (viewing it as a subgroup of $\operatorname{Gal}\left(F_{p^{k}}, \mathbb{Q}(j)\right)$ ). From now on, we will indicate the cusp $\sigma^{-1}(\infty)$ simply by $\sigma^{-1}$.

We may also index the primitive Siegel function by elements of the Cartan Group. Following [7], if $\alpha \in C\left(p^{k}\right) /\{ \pm I\}$ we put:

$$
g_{\alpha}=g_{e_{1} \alpha} \text { where } e_{1}=\left(\frac{1}{p^{k}}, 0\right) .
$$

It should be noted that $g_{\alpha}$ is defined up to a root of unity (this follows from Proposition 4.1, second claim). Nonetheless, $g_{\alpha}^{12 p^{k}}$ is univocally defined as well as its divisor:

Proposition 4.5. We have:

$$
\operatorname{div} g_{\alpha}^{12 p^{k}}=6 p^{2 k} \sum_{\beta \in C\left(p^{k}\right) /\{ \pm I\}} B_{2}\left(\left\langle\frac{T\left(\alpha \beta^{-1}\right)}{p^{k}}\right\rangle\right) \beta
$$

where the map $T$ on $2 \times 2$ matrices is defined as follows:

$$
T:\left(\begin{array}{ll}
a & b \\
c & d
\end{array}\right) \mapsto a .
$$

Proof. See [7, Paragraph 5.1]

The first part of [7] culminates with the theorem below. The computation of the order of the cuspidal divisor class group on $X\left(p^{k}\right)$ could be considered analogous to that in the study of cyclotomic fields: instead of the generalized Bernoulli numbers $B_{1, \chi}$ encountered in the latter case, in the former we will define the second generalized Bernoulli numbers $B_{2, \chi}$.

Theorem 4.6. Let $p$ a prime $\geq 5$. Let $R:=\mathbb{Z}\left[C\left(p^{k}\right) /\{ \pm 1\}\right]$ and $R_{0}$ the ideal of $R$ consisting of elements of degree 0. The Cuspidal Divisor Class Group $\mathfrak{C}_{p^{k}}$ is an $R$-module, more precisely there exists a Stickelberger element

$$
\theta=\frac{p^{k}}{2} \sum_{\beta \in C\left(p^{k}\right) /\{ \pm 1\}} B_{2}\left(\left\langle\frac{T(\beta)}{p^{k}}\right\rangle\right) \beta^{-1} \in \mathbb{Q}\left[C\left(p^{k}\right) /\{ \pm 1\}\right]
$$

such that:

$$
\mathfrak{C}_{p^{k}} \cong R_{0} / R \cap R \theta .
$$

For any character $\chi$ of $C\left(p^{k}\right) /\{ \pm I\}$ (identified with an even character of $C\left(p^{k}\right)$ ) we let:

$$
B_{2, \chi}=\sum_{\alpha \in C\left(p^{k}\right) /\{ \pm I\}} B_{2}\left(\left\langle\frac{T(\alpha)}{p^{k}}\right\rangle\right) \chi(\alpha) .
$$


The order of the cuspidal divisor class group on $X\left(p^{k}\right)$ is:

$$
\left|\mathfrak{C}_{p^{k}}\right|=\frac{12 p^{3 k}}{\left|C\left(p^{k}\right)\right|} \prod_{\chi \neq 1} \frac{p^{k}}{2} B_{2, \chi} .
$$

Proof. [7, Chapter 5].

\section{Non-split Cartan Groups}

Following [1] or [15, pag. 194], let $n$ a positive integer and let $A$ be a finite free commutative algebra of rank 2 over $\mathbb{Z} / n \mathbb{Z}$ with unit discriminant. Fixing a basis for $A$ we can use the action of $A^{*}$ on $A$ to embed $A^{*}$ in $G L_{2}(\mathbb{Z} / n \mathbb{Z})$. If for every prime $p \mid n$ the $\mathbb{F}_{p}$ algebra $A / p A$ is isomorphic to $\mathbb{F}_{p^{2}}$, the image of $A^{*}$ just now described is called a non-split Cartan subgroup of $G L_{2}(\mathbb{Z} / n \mathbb{Z})$. Therefore, such a group $G$ has the property that for every prime $p$ dividing $n$ the reduction of $G \bmod p$ is isomorphic to $\mathbb{F}_{p^{2}}$. All the non-split Cartan subgroups of $G L_{2}(\mathbb{Z} / n \mathbb{Z})$ are conjugate and so are their normalizers.

In this paper we are interested in the case $n=p^{k}$ and $p \neq 2,3$. The cases $p=2$ and $p=3$ are essentially equal but require more cumbersome calculations (see [7, Theorem 5.3, Chapter 3] and 7, Theorem 1.3, Chapter 4]). Choose a squarefree integer $\epsilon \equiv 3 \bmod 4$ and such that its reduction modulo $p$ is a quadratic non-residue. If $p \equiv 3 \bmod 4$, a canonical choice could be $\epsilon=-1$. Let $K=\mathbb{Q}(\sqrt{\epsilon})$ and $\mathbf{O}_{K}=\mathbb{Z}[\sqrt{\epsilon}]$ its ring of integers. After choosing a basis for $\mathbf{O}_{K}$ over $\mathbb{Z}$ we can represent any element of $\left(\mathbf{O}_{K} / p^{k} \mathbf{O}_{K}\right)^{*}$ with its corresponding multiplication matrix in $G L_{2}\left(\mathbb{Z} / p^{k} \mathbb{Z}\right)$ with respect to the chosen basis. This embedding produces a non-split Cartan subgroup of $G L_{2}\left(\mathbb{Z} / p^{k} \mathbb{Z}\right)$ and we will denote it by $C_{n s}\left(p^{k}\right)$. Notice that such a group is isomorphic to the already introduced $C\left(p^{k}\right)$.

To describe the normalizer $C_{n s}^{+}\left(p^{k}\right)$ of $C_{n s}\left(p^{k}\right)$ in $G L_{2}\left(\mathbb{Z} / p^{k} \mathbb{Z}\right)$ it will suffice to consider the following group automorphism induced by conjugation by a fixed $c \in C_{n s}^{+}\left(p^{k}\right)$ :

$$
\begin{gathered}
\phi_{c}: C_{n s}\left(p^{k}\right) \longrightarrow C_{n s}\left(p^{k}\right) \\
x \longmapsto \phi_{c}(x)=c x c^{-1} .
\end{gathered}
$$

The group automorphism $\phi_{c}$ extends to a ring automorphism of $\left(\mathbf{O}_{K} / p^{k} \mathbf{O}_{K}\right) \cong$ $\left(\mathbb{Z} / p^{k} \mathbb{Z}\right)[\sqrt{\epsilon}]$ so if $\phi_{c}$ is not the trivial automorphism we necessarily have $\phi_{c}(\sqrt{\epsilon})=-\sqrt{\epsilon}$. 
Proposition 5.1. If $p \neq 2$ we have the following isomorphism:

$$
\begin{gathered}
C_{n s}\left(p^{k}\right) \simeq \mathbb{Z} / p^{k-1} \mathbb{Z} \times \mathbb{Z} / p^{k-1} \mathbb{Z} \times \mathbb{Z} /\left(p^{2}-1\right) \mathbb{Z}, \\
C_{n s}^{+}\left(p^{k}\right) \simeq\left(\mathbb{Z} / p^{k-1} \mathbb{Z} \times \mathbb{Z} / p^{k-1} \mathbb{Z} \times \mathbb{Z} /\left(p^{2}-1\right) \mathbb{Z}\right) \rtimes_{\phi} \mathbb{Z} / 2 \mathbb{Z} .
\end{gathered}
$$

Proof. Let $a_{1}+\sqrt{\epsilon} a_{2} \in\left(\mathbf{O}_{K} / p^{k} \mathbf{O}_{K}\right)$ : it is invertible if and only if $\left(a_{1}, a_{2}\right)$ is primitive or in other words $p$ does not divide both $a_{1}$ and $a_{2}$ so we have $\left|C_{n s}\left(p^{k}\right)\right|=p^{2 k-2}\left(p^{2}-1\right)$. Consider the reduction $\bmod p$ :

$$
\begin{gathered}
C_{n s}\left(p^{k}\right) \longrightarrow \mathbb{F}_{p^{2}}^{*} \\
a_{1}+\sqrt{\epsilon} a_{2} \longmapsto \overline{a_{1}}+\sqrt{\epsilon} \overline{a_{2}} .
\end{gathered}
$$

The map is surjective and let $B$ its kernel:

$$
B:=\left\{x \in\left(\mathbf{O}_{K} / p^{k} \mathbf{O}_{K}\right)^{*} \text { such that } x \equiv 1 \bmod p\right\}
$$

$|B|=p^{2 k-2}$ : it remains to check that $B \simeq \mathbb{Z} / p^{k-1} \mathbb{Z} \times \mathbb{Z} / p^{k-1} \mathbb{Z}$. Let $k \geq 2$ and $p \neq 2$. First, we check that for all $x \in \mathbf{O}_{K}$ we have $(1+x p)^{p^{k-2}} \equiv 1+x p^{k-1}$ $\bmod p^{k}$. In case $k=2$ there is nothing to prove. We proceed by induction on $k$ : suppose the claim is true for some $k \geq 2$. We have:

$$
\begin{gathered}
(1+x p)^{p^{k-2}}=1+x p^{k-1}+y p^{k} \\
(1+x p)^{p^{k-1}}=\sum_{j=0}^{p}\left(\begin{array}{c}
p \\
j
\end{array}\right)\left(1+x p^{k-1}\right)^{p-j}\left(y p^{k}\right)^{j} \equiv\left(1+x p^{k-1}\right)^{p} \quad \bmod p^{k+1}, \\
\left(1+x p^{k-1}\right)^{p}=\sum_{j=0}^{p}\left(\begin{array}{c}
p \\
j
\end{array}\right)\left(x p^{k-1}\right)^{j} \equiv 1+x p^{k} \quad \bmod p^{k+1} .
\end{gathered}
$$

In conclusion: $(1+x p)^{p^{k-1}} \equiv 1+x p^{k} \bmod p^{k+1}$. From the previous claim follows that if $h \leq k-1$ is such that $x \in p^{h} \mathbf{O}_{K} \backslash p^{h+1} \mathbf{O}_{K}$ then the reduction of $1+x p$ in $B$ has order $p^{k-1-h}$. So $B$ has $p^{2 k-2}-p^{2 k-4}$ elements of order $p^{k-1}$ and the proposition is proved. The second isomorphism follows immediately.

We present now the modular curves $X_{n s}(n)$ and $X_{n s}^{+}(n)$ associated to the subgroups $C_{n s}(n)$ and $C_{n s}^{+}(n)$. First of all, $Y(n)$ (the non-cuspidal points of $X(n))$ are isomorphism classes of pairs $(E,(P, Q))$ where $E$ is a complex elliptic curve and $(P, Q)$ constitute a $\mathbb{Z} / n \mathbb{Z}$-basis of the $n$-torsion subgroup $E[n]$ with $e_{n}(P, Q)=e^{2 \pi i / n}$ where $e_{n}$ is the Weil pairing discussed in details in [4, Chapter 7]. By definition, two pairs $(E,(P, Q))$ and $\left(E^{\prime},\left(P^{\prime}, Q^{\prime}\right)\right)$ are considered equivalent in $Y(n)$ if and only if there exists an isomorphism 
between $E$ and $E^{\prime}$ taking $P$ to $P^{\prime}$ and $Q$ to $Q^{\prime}$. Notice that the definition is well-posed since the Weil pairing is invariant under isomorphism, i.e. if $f: E \rightarrow E^{\prime}$ is an isomorphism of elliptic curves and $e_{n}^{\prime}$ is the Weil pairing on $E^{\prime}$ we have:

$$
e_{n}^{\prime}(f(P), f(Q))=e_{n}(P, Q)
$$

Since $G L_{2}(\mathbb{Z} / n \mathbb{Z})$ acts on $E[n]$ and since for every $\gamma \in G L_{2}(\mathbb{Z} / n \mathbb{Z})$ we have $e_{n}(\gamma(P, Q))=e_{n}(P, Q)^{\operatorname{det} \gamma}$, the group $S L_{2}(\mathbb{Z} / n \mathbb{Z})$ acts on $Y(n)$ on the right in the following way:

$$
\left(\begin{array}{ll}
a & b \\
c & d
\end{array}\right) \cdot(E,(P, Q))=(E,(a P+c Q, b P+d Q)) .
$$

Define:

$$
\begin{aligned}
& C_{n s}^{\prime}(n):=C_{n s}(n) \cap S L_{2}(\mathbb{Z} / n \mathbb{Z}), \\
& C_{n s}^{\prime+}(n):=C_{n s}^{+}(n) \cap S L_{2}(\mathbb{Z} / n \mathbb{Z}),
\end{aligned}
$$

$\Gamma_{n s}(n):=\left\{M \in S L_{2}(\mathbb{Z})\right.$ such that $M \equiv M^{\prime} \bmod n$ for some $\left.M^{\prime} \in C_{n s}^{\prime}(n)\right\}$, $\Gamma_{n s}^{+}(n):=\left\{M \in S L_{2}(\mathbb{Z})\right.$ such that $M \equiv M^{\prime} \bmod n$ for some $\left.M^{\prime} \in C_{n s}^{\prime+}(n)\right\}$.

A possible explicit description for these groups is:

$$
\begin{gathered}
C_{n s}\left(p^{k}\right)=\left\{M_{s}=\left(\begin{array}{cc}
a & b \\
\epsilon b & a
\end{array}\right) \in G L_{2}\left(\mathbb{Z} / p^{k} \mathbb{Z}\right) \text { with } s=a+\sqrt{\epsilon} b \in\left(\mathbf{O}_{K} / p^{k} \mathbf{O}_{K}\right)^{*}\right\}, \\
C_{n s}^{+}\left(p^{k}\right)=\left\langle\left(\begin{array}{cc}
a & b \\
\epsilon b & a
\end{array}\right) \in C_{n s}\left(p^{k}\right), C=\left(\begin{array}{cc}
1 & 0 \\
0 & -1
\end{array}\right)\right\rangle .
\end{gathered}
$$

If $s \in\left(\mathbf{O}_{K} / p^{k} \mathbf{O}_{K}\right)^{*}$ we define $|s|:=s \bar{s} \in\left(\mathbb{Z} / p^{k} \mathbb{Z}\right)^{*}$ where $\bar{s}$ is the conjugate of $s$. So we have:

$$
\begin{aligned}
& C_{n s}^{\prime}\left(p^{k}\right)=\left\{M_{s}=\left(\begin{array}{cc}
a & b \\
\epsilon b & a
\end{array}\right) \in C_{n s}\left(p^{k}\right) \text { such that }|s|=|a+\sqrt{\epsilon} b|=1 \bmod p^{k}\right\}, \\
& C_{n s}^{\prime+}\left(p^{k}\right)=C_{n s}^{\prime}\left(p^{k}\right) \cup\left\{M_{s} C=\left(\begin{array}{cc}
a & -b \\
\epsilon b & -a
\end{array}\right) \text { with }|s|=|a+\sqrt{\epsilon} b|=-1 \bmod p^{k}\right\} .
\end{aligned}
$$

Points in $Y_{n s}(n)$ are nothing but orbits of $Y(n)$ under the action of $C_{n s}^{\prime}(n)$ and similarly for $Y_{n s}^{+}(n)$ and $C_{n s}^{\prime+}(n)$. The above-mentioned action extends uniquely to $X(n)$. The quotients $X_{n s}(n)$ and $X_{n s}^{+}(n)$ are isomorphic as Riemann surfaces to $\mathbb{H}^{*} / \Gamma_{n s}(n)$ and $\mathbb{H}^{*} / \Gamma_{n s}^{+}(n)$ respectively.

Using the identification of the cusps of $X\left(p^{k}\right)$ with the elements of $C\left(p^{k}\right) /\{ \pm I\}$ explained in the previous section we obtain a shorter proof of the first claim of [1, Proposition 7.10]: 
Proposition 5.2. We identify the cusps of $X_{n s}\left(p^{k}\right)$ with $\left(\mathbb{Z} / p^{k} \mathbb{Z}\right)^{*}$ and the cusps of $X_{n s}^{+}\left(p^{k}\right)$ with $H=\left(\mathbb{Z} / p^{k} \mathbb{Z}\right)^{*} /\{ \pm 1\}$. So $X_{n s}\left(p^{k}\right)$ has $p^{k-1}(p-1)$ cusps and $X_{n s}^{+}\left(p^{k}\right)$ has $p^{k-1} \frac{p-1}{2}$ cusps.

Proof. We identify the cusps of $X\left(p^{k}\right)$ with the elements of $C\left(p^{k}\right) /\{ \pm I\} \cong$ $C_{n s}\left(p^{k}\right) /\{ \pm I\} \cong\left(\mathbf{O}_{K} / p^{k} \mathbf{O}_{K}\right)^{*} /\{ \pm 1\}$. Bearing this in mind, it is clear that $\pm M_{r}, \pm M_{r^{\prime}} \in C_{n s}\left(p^{k}\right) /\{ \pm I\}$ represent the same cusp in $X_{n s}\left(p^{k}\right)$ if and only if there exists $s \in\left(\mathbf{O}_{K} / p^{k} \mathbf{O}_{K}\right)^{*}$ with $|s|=1$ such that $\pm r= \pm s r^{\prime}$. But this is equivalent to say that $|r|=\left|s r^{\prime}\right|=\left|r^{\prime}\right| \operatorname{or} \operatorname{det} M_{r}=\operatorname{det} M_{r^{\prime}} \bmod p^{k}$ and consequently we may identify the cusps of $X_{n s}\left(p^{k}\right)$ with $\left(\mathbb{Z} / p^{k} \mathbb{Z}\right)^{*}$. For the same reason $\pm M_{r}, \pm M_{r^{\prime}} \in C_{n s}\left(p^{k}\right) /\{ \pm I\}$ are indistinguishable in $X_{n s}^{+}\left(p^{k}\right)$ if and only if they were already indistinguishable in $X_{n s}\left(p^{k}\right)$ or there exists $s^{\prime} \in\left(\mathbf{O}_{K} / p^{k} \mathbf{O}_{K}\right)^{*}$ with $\left|s^{\prime}\right|=-1$ such that $\pm r= \pm s^{\prime} \overline{r^{\prime}}$ that is equivalent to say $|r|=\left|s^{\prime} \bar{r}^{\prime}\right|=-\left|r^{\prime}\right|$ or $\operatorname{det} M_{r}=-\operatorname{det} M_{r^{\prime}} \bmod p^{k}$. In conclusion we may identify the cusps of $X_{n s}^{+}\left(p^{k}\right)$ with $H=\left(\mathbb{Z} / p^{k} \mathbb{Z}\right)^{*} /\{ \pm 1\}$.

Furthermore, we can deduce that the covering $\pi: X_{n s}\left(p^{k}\right) \rightarrow X\left(p^{k}\right)$ is not ramified above the cusps. So the ramification degree of a cusp of $X_{n s}\left(p^{k}\right)$ under the covering projection $\pi^{\prime}: X_{n s}\left(p^{k}\right) \rightarrow S L_{2}(\mathbb{Z}) \backslash \mathbb{H}^{*}$, is equal to the one of a cusp of $X\left(p^{k}\right)$ respect to $\pi^{\prime \prime}: X\left(p^{k}\right) \rightarrow S L_{2}(\mathbb{Z}) \backslash \mathbb{H}^{*}$ that is $p^{k}$. The same happens for $X_{n s}^{+}\left(p^{k}\right)$.

\section{Modular units on non-split Cartan curves}

Let $t \in\left(\left(\mathbf{O}_{K} / p^{k} \mathbf{O}_{K}\right)^{*} /\{ \pm 1\}\right)$ : write it in the form $t=a_{1}+\sqrt{\epsilon} a_{2}$ choosing $a_{1}, a_{2} \in \mathbb{Z}$ such that $0 \leq a_{1} \leq \frac{p^{k}-1}{2}, 0 \leq a_{2} \leq p^{k}-1$ and $a_{2} \leq \frac{p^{k}-1}{2}$ if $a_{1}=0$. Define:

$$
[t]:=\frac{1}{p^{k}}\left(a_{1}, a_{2}\right) \text {. }
$$

If $s \in\left(\mathbf{O}_{K} / p^{k} \mathbf{O}_{K}\right)^{*}$ we define $[s]:=[\{ \pm s\}]$. Notice that if $s, t \in\left(\mathbf{O}_{K} / p^{k} \mathbf{O}_{K}\right)^{*}$, $|s|=1$ and $\gamma_{s} \in \Gamma_{n s}\left(p^{k}\right)$ lifts $M_{s}$ we have:

$$
[t] \gamma_{s}-[t s] \in \mathbb{Z}^{2} \text { or }[t] \gamma_{s}+[t s] \in \mathbb{Z}^{2} .
$$

Analogously if $|s|=-1$ and $\gamma$ lifts $M_{s} C$ to $\Gamma_{n s}^{+}\left(p^{k}\right)$ we have:

$$
[t] \gamma-[\overline{t s}] \in \mathbb{Z}^{2} \text { or }[t] \gamma+[\overline{t s}] \in \mathbb{Z}^{2} .
$$

These relations together with Proposition 4.1 imply: 
Proposition 6.1. The Klein forms: $\mathfrak{k}_{[t] \gamma_{s}}(\tau)$ and $\mathfrak{k}_{[t s]}(\tau)$ up to a $2 p^{k}$-th root of unity represent the same function in the sense that:

$$
\mathfrak{k}_{[t] \gamma_{s}}(\tau)=c \mathfrak{k}_{[t s]}(\tau)
$$

for some $c \in \boldsymbol{\mu}_{\mathbf{2} p^{k}}$. Similarly, for the Klein forms $\mathfrak{k}_{[t] \gamma}(\tau)$ and $\mathfrak{k}_{[\overline{t s}]}(\tau)$ we have:

$$
\mathfrak{k}_{[t] \gamma}(\tau)=c^{\prime} \mathfrak{k}_{[\overline{t s}]}(\tau)
$$

for some $c^{\prime} \in \boldsymbol{\mu}_{\mathbf{2} p^{k}}$.

For $h \in\left(\mathbb{Z} / p^{k} \mathbb{Z}\right)^{*}$ we define the following complex-valued functions on $\mathbb{H}$ :

$$
\begin{gathered}
T_{h}(\tau):=\prod_{t \in\left(\left(\mathbf{O}_{K} / p^{k} \mathbf{O}_{K}\right)^{*} /\{ \pm 1\}\right),|t|=h} \mathfrak{k}_{[t]}(\tau), \\
G_{h}(\tau):=T_{h}(\tau)(\Delta(\tau))^{p^{k-1} \frac{p+1}{24}}=\prod_{t \in\left(\left(\mathbf{O}_{K} / p^{k} \mathbf{O}_{K}\right)^{*} /\{ \pm 1\}\right),|t|=h} g_{[t]}(\tau) .
\end{gathered}
$$

For $h \in\left(\mathbb{Z} / p^{k} \mathbb{Z}\right)^{*} /\{ \pm 1\}$ consider:

$$
\begin{gathered}
T_{h}^{+}(\tau):=\prod_{t \in\left(\left(\mathbf{O}_{K} / p^{k} \mathbf{O}_{K}\right)^{*} /\{ \pm 1\}\right), \pm|t|=h} \mathfrak{k}_{[t]}(\tau), \\
G_{h}^{+}(\tau):=T_{h}^{+}(\tau)(\Delta(\tau))^{p^{k-1} \frac{p+1}{12}}=\prod_{t \in\left(\left(\mathbf{O}_{K} / p^{k} \mathbf{O}_{K}\right)^{*} /\{ \pm 1\}\right), \pm|t|=h} g_{[t]}(\tau) .
\end{gathered}
$$

Proposition 6.2. Let $p \neq 2,3$ a prime. Consider:

$$
g(\tau)=\prod_{\left.x \in\left(\left(\mathbf{O}_{K} / p^{k} \mathbf{O}_{K}\right)^{*} /\{ \pm 1\}\right)\right)} g_{[x]}^{m(x)}(\tau)
$$

and suppose that it is a modular unit on $X\left(p^{k}\right)$ (or equivalently that it satisfies the conditions of Theorem 4.3). If $g(\tau)$ is a modular unit on $X_{n s}\left(p^{k}\right)$ there exist integers $\left\{n_{h}\right\}_{h \in\left(\mathbb{Z} / p^{k} \mathbb{Z}\right)^{*}}$ such that:

$$
g(\tau)=\prod_{h \in\left(\mathbb{Z} / p^{k} \mathbb{Z}\right)^{*}} G_{h}^{n_{h}}(\tau) .
$$

Similarly, if the function $g(\tau)$ is a modular unit on $X_{n s}^{+}\left(p^{k}\right)$, there exist integers $\left\{n_{h}^{+}\right\}_{h \in\left(\mathbb{Z} / p^{k} \mathbb{Z}\right)^{*} /\{ \pm 1\}}$ such that:

$$
g(\tau)=\prod_{h \in\left(\left(\mathbb{Z} / p^{k} \mathbb{Z}\right)^{*} /\{ \pm 1\}\right)} G_{h}^{+n_{h}^{+}}(\tau) .
$$


Proof. We look for conditions on the exponents $\{m(x)\}_{x \in\left(\left(\mathbf{O}_{K} / p^{k} \mathbf{O}_{K}\right)^{*} /\{ \pm 1\}\right)}$ that guarantee:

$$
\frac{g\left(\sigma^{-1}(\tau)\right)}{g(\tau)} \in \mathbb{C} \text { for every } \sigma \in \Gamma_{n s}\left(p^{k}\right)\left(\text { respectively } \Gamma_{n s}^{+}\left(p^{k}\right)\right) .
$$

From Proposition 4.1, assertion (3), the fact that $\Delta(\tau)$ is weakly modular of weight 12 and that by hypotesis 12 divides $\sum m(x)$ we have:

$$
\begin{aligned}
g\left(\sigma^{-1}(\tau)\right) & =\left(\Delta\left(\sigma^{-1}(\tau)\right)\right)^{\frac{1}{12} \sum m(x)} \prod \mathfrak{k}_{[x]}^{m(x)}\left(\sigma^{-1}(\tau)\right)= \\
& =(\Delta(\tau))^{\frac{1}{12} \sum m(x)} \prod \mathfrak{k}_{[x] \sigma^{-1}}^{m(x)}(\tau) .
\end{aligned}
$$

By Proposition 5.1, $C_{n s}^{\prime}\left(p^{k}\right)$ is a cyclic group with $(p+1) p^{k-1}$ elements. Let $M_{r}$ be a generator where $r$ is a generator of:

$$
\left\{s \in\left(\mathbf{O}_{K} / p^{k} \mathbf{O}_{K}\right)^{*} \text { with }|s|=1\right\} .
$$

Every $S \in C_{n s}^{\prime+}\left(p^{k}\right) \backslash C_{n s}^{\prime}\left(p^{k}\right)$ is of the form $M_{t} C$ where $t \in\left(\mathbf{O}_{K} / p^{k} \mathbf{O}_{K}\right)^{*}$ and $|t|=-1$. Fix $S$ and choose $\gamma_{r}$ lifting $M_{r}$ in $\Gamma_{n s}\left(p^{k}\right)$ and $\gamma_{t}$ lifting $M_{t}$ in $G L_{2}(\mathbb{Z})$ with $\operatorname{det} \gamma_{t}=-1$. Of course $\gamma_{t} C$ lifts $S$ in $\Gamma_{n s}^{+}\left(p^{k}\right)$.

For every $j$ we have that:

$$
\begin{gathered}
\left(\left([x] \bmod \mathbb{Z}^{2}\right) /\{ \pm 1\}\right) \longmapsto\left(\left(\left[x r^{j}\right] \bmod \mathbb{Z}^{2}\right) /\{ \pm 1\}\right) \text { and } \\
\left(\left([x] \bmod \mathbb{Z}^{2}\right) /\{ \pm 1\}\right) \longmapsto\left(\left(\left[\overline{x r^{j} t}\right] \bmod \mathbb{Z}^{2}\right) /\{ \pm 1\}\right)
\end{gathered}
$$

are permutations of the primitive elements in $\left(\left(\frac{1}{p^{k}} \mathbb{Z}\right)^{2} \bmod \mathbb{Z}^{2}\right) /( \pm 1)$. As a consequence of these observations and Proposition [6.1, taking $\sigma=\left(\gamma_{r}\right)^{j}$ we have:

$$
\begin{gathered}
(\Delta(\tau))^{\frac{1}{12} \sum m(x)} \prod \mathfrak{k}_{[x] \gamma_{r}^{-j}}^{m(x)}(\tau)=(\Delta(\tau))^{\frac{1}{12} \sum m(x)} \prod \mathfrak{k}_{\left[x r^{j}\right] \gamma_{r}^{-j}}^{m(\tau)}(\tau)= \\
=c_{j}(\Delta(\tau))^{\frac{1}{12} \sum m(x)} \prod \mathfrak{k}_{[x]}^{m\left(x r^{j}\right)}(\tau)=c_{j} \prod g_{[x]}^{m\left(x r^{j}\right)}(\tau),
\end{gathered}
$$

where $\left\{c_{j}\right\}_{j}$ are $2 p^{k}$-th roots of unity. Taking $\sigma=\left(\gamma_{r}\right)^{j} \gamma_{t} C$ we obtain:

$$
\begin{gathered}
(\Delta(\tau))^{\frac{1}{12} \sum m(x)} \prod \mathfrak{k}_{[x] C \gamma_{t}^{-1} \gamma_{r}^{-j}}^{m(x)}(\tau)=(\Delta(\tau))^{\frac{1}{12} \sum m(x)} \prod \mathfrak{k}_{\left[\overline{x r^{j} t}\right] C \gamma_{t}^{-1} \gamma_{r}^{-j}}^{m\left(\overline{r^{j}}\right)}(\tau)= \\
=d_{j}(\Delta(\tau))^{\frac{1}{12} \sum m(x)} \prod \mathfrak{k}_{[x]}^{m\left(\overline{x r^{j} t}\right)}(\tau)=d_{j} \prod g_{[x]}^{m\left(\overline{x r^{j} t}\right)}(\tau),
\end{gathered}
$$

where $\left\{d_{j}\right\}_{j}$ are $2 p^{k}$-th roots of unity. Consider the following expression:

$$
\frac{g\left(\gamma_{r}^{-1}(\tau)\right)}{g(\tau)}=c_{1} \prod g_{[x]}^{m(x r)-m(x)}(\tau)
$$


By the independence of Siegel functions [7, p.42 or p.120] a product $\prod g_{[x]}^{l(x)}$ is constant if and only if the exponents $l(x)$ are all equal. So the previous quotient is constant if and only if:

$$
a\left(x r^{j}\right)=m\left(x r^{j+1}\right)-m\left(x r^{j}\right) \text { satisfy } a\left(x r^{j}\right)=a\left(x r^{l}\right) \text { for all } j, l \in \mathbb{Z} .
$$

But $\left(\gamma_{r}\right)^{\frac{p+1}{2} p^{k-1}} \equiv-I \bmod p^{k}$ and $r^{\frac{p+1}{2} p^{k-1}}=-1 \bmod p^{k}$. So we have that $\sum_{j=1}^{\frac{p+1}{2} p^{k-1}} a\left(x r^{j}\right)=0$ and consequently $a\left(x r^{j}\right)=0$ for every $j$, which implies that $m\left(x r^{j}\right)$ does not depend on $j$. Since $g(\tau)$ is $\Gamma\left(p^{k}\right)$-invariant and every element in $\Gamma_{n s}\left(p^{k}\right)$ can be written in the form $\gamma \gamma_{r}^{j}$ with $\gamma \in \Gamma\left(p^{k}\right)$, we conclude that if $g\left(\sigma^{-1}(\tau)\right) / g(\tau) \in \mathbb{C}$ for every $\sigma \in \Gamma_{n s}\left(p^{k}\right)$, this implies that if $|x|=|y|$ then $m(x)=m(y)$. For each $h$ invertible mod $p^{k}$ choose $x$ with $|x|=h$, put $n_{h}:=m(x)$ and the first claim follows.

Consider now:

$$
\frac{g\left(\left(C \gamma_{t}^{-1}\right)(\tau)\right)}{g(\tau)}=d_{0} \prod g_{[x]}^{m(\bar{x})-m(x)}(\tau) .
$$

If this quotient is constant the exponent of $g_{[x]}(\tau)$ is equal to the exponent of the Siegel function $g_{[\bar{x} r t]}(\tau)$. So:

$$
m(\overline{x t})-m(x)=m\left(x \overline{r t^{2}}\right)-m(\bar{x} r t)
$$

or equivalently: $m(\overline{x t})+m(\bar{x} r t)=m(x)+m\left(x \overline{r t^{2}}\right)$. But $\left|r t \overline{t^{-1}}\right|=1$ so $m(\overline{x t})=m(\bar{x} r t)$ and $\left|\overline{r t^{2}}\right|=1$ so $m(x)=m\left(x \overline{r t^{2}}\right)$. Hence $m(x)=m(\overline{x t})$ and observe that $|x|=-|\overline{x t}|$. So, in consideration of the previous result, we can conclude that $g\left(\sigma^{-1}(\tau)\right) / g(\tau) \in \mathbb{C}$ for every $\sigma \in \Gamma_{n s}^{+}\left(p^{k}\right)$ implies that if $|x|=|y|$ or $|x|=-|y|$ then $m(x)=m(y)$. For every $h \in\left(\mathbb{Z} / p^{k} \mathbb{Z}\right)^{*} /\{ \pm 1\}$ choose $x$ such that $\pm|x|=h$ and define $n_{h}^{+}:=m(x)$ and the second claim follows.

Proposition 6.3. The product:

$$
\prod_{h \in\left(\mathbb{Z} / p^{k} \mathbb{Z}\right)^{*}} T_{h}^{n_{h}}(\tau)
$$

is a nearly holomorphic modular form for $\Gamma\left(p^{k}\right)$ if and only if $p$ divides $\sum_{h} n_{h} h$.

Proof. First of all, for every $h$ invertible $\bmod p^{k}$ :

$$
\sum_{\substack{ \pm s \in\left(\left(\mathbf{O}_{K} / p^{k} \mathbf{O}_{K}\right)^{*} /\{ \pm 1\}\right) \\| \pm s|=h}}\left(\frac{1}{2}(s+\bar{s})\right)^{2}=\frac{h}{4}(p+1) p^{k-1} \bmod p^{k},
$$


(2)

$$
\begin{gathered}
\sum_{\substack{ \pm s \in\left(\left(\mathbf{O}_{K} / p^{k} \mathbf{O}_{K}\right)^{*} /\{ \pm 1\}\right) \\
| \pm s|=h}}\left(\frac{1}{2 \sqrt{\epsilon}}(s-\bar{s})\right)^{2}=-\frac{h}{4 \epsilon}(p+1) p^{k-1} \bmod p^{k} \\
\sum_{\substack{\left. \pm s \in\left(\mathbf{O}_{K} / p^{k} \mathbf{O}_{K}\right)^{*} /\{ \pm 1\}\right) \\
| \pm s|=h}}\left(\frac{1}{2}(s+\bar{s})\right)\left(\frac{1}{2 \sqrt{\epsilon}}(s-\bar{s})\right)=0 \quad \bmod p^{k} \\
\hline
\end{gathered}
$$

We prove only the first assertion because the other statements can be shown by the same argument. Every $s \in\left(\mathbf{O}_{K} / p^{k} \mathbf{O}_{K}\right)^{*}$ with $|s|=h$ can be written as $s=r^{i} \alpha_{h}$ where $r$ is a generator of the subgroup $\{t \in$ $\left(\mathbf{O}_{K} / p^{k} \mathbf{O}_{K}\right)^{*}$ with $\left.|t|=1\right\}$ and $\alpha_{h}$ are fixed elements such that $\left|\alpha_{h}\right|=h$.

$$
\begin{gathered}
\sum_{| \pm s|=h}\left(\frac{1}{2}(s+\bar{s})\right)^{2}=\sum_{i=0}^{\frac{p+1}{2} p^{k-1}-1}\left(\frac{1}{2}\left(r^{i} \alpha_{h}+\overline{r^{i} \alpha_{h}}\right)\right)^{2}= \\
=\frac{\alpha_{h}^{2}}{4} \sum_{i=0}^{\frac{p+1}{2} p^{k-1}-1}\left(r^{2 i}\right)+\frac{\overline{\alpha_{h}^{2}}}{4} \sum_{i=0}^{\frac{p+1}{2} p^{k-1}-1}\left(r^{-2 i}\right)+\frac{\alpha_{h} \overline{\alpha_{h}}}{4}(p+1) p^{k-1}
\end{gathered}
$$

and the assertion (1) follows because:

$$
\sum_{i=0}^{\frac{p+1}{2} p^{k-1}-1} r^{2 i}=\sum_{i=0}^{\frac{p+1}{2} p^{k-1}-1} r^{-2 i}=\frac{1-r^{(p+1) p^{k-1}}}{1-r^{2}}=0 \quad \bmod p^{k}
$$

To prove this proposition we apply Proposition 4.1 to the product $\prod_{h} T_{h}^{n_{h}}(\tau)$. Considering that for every $s=a_{1}+\sqrt{\epsilon} a_{2} \in\left(\mathbf{O}_{K} / p^{k} \mathbf{O}_{K}\right)^{*}$ we have:

$$
\begin{gathered}
p^{k}[s] \equiv\left(a_{1}, a_{2}\right) \bmod \left(p^{k} \mathbb{Z}\right)^{2} \text { or } p^{k}[s] \equiv-\left(a_{1}, a_{2}\right) \bmod \left(p^{k} \mathbb{Z}\right)^{2} \text { and: } \\
\left(a_{1}, a_{2}\right) \equiv\left(\frac{1}{2}(s+\bar{s}), \frac{1}{2 \sqrt{\epsilon}}(s-\bar{s})\right) \bmod \left(p^{k} \mathbb{Z}\right)^{2}
\end{gathered}
$$

and reformulating condition (5) of Proposition 4.1 in terms of assertions (1),(2) and (3) we attain the desired result.

From this proposition it follows immediately that the functions $T_{h}^{+}(\tau)$ are nearly holomorphic for $\Gamma\left(p^{k}\right)$. We will examine them further in details.

For every $s=\left(\begin{array}{ll}a & b \\ c & d\end{array}\right) \in S L_{2}(\mathbb{Z})$ define:

$$
J_{s}(\tau)=(c \tau+d)^{-(p+1) p^{k-1}}, \tau \in \mathbb{H} .
$$


Proposition 6.4. For every prime $p \equiv 3 \bmod 4$, for every $h \in\left(\left(\mathbb{Z} / p^{k} \mathbb{Z}\right)^{*} /\{ \pm 1\}\right)$ and for every $s \in \Gamma_{n s}^{+}\left(p^{k}\right)$ we have:

$$
T_{h}^{+}(s(\tau))=J_{s}(\tau) T_{h}^{+}(\tau)
$$

in other words $T_{h}^{+}(\tau)$ is a nearly holomorphic modular form for $\Gamma_{n s}^{+}\left(p^{k}\right)$ of weight $-(p+1) p^{k-1}$.

If $p \equiv 1 \bmod 4$ and $s \in \Gamma_{n s}\left(p^{k}\right)$ we have:

$$
T_{h}^{+}(s(\tau))=J_{s}(\tau) T_{h}^{+}(\tau)
$$

in other words $T_{h}^{+}(\tau)$ is a nearly holomorphic modular form for $\Gamma_{n s}\left(p^{k}\right)$ of weight $-(p+1) p^{k-1}$.

If $p \equiv 1 \bmod 4$ and $s \in \Gamma_{n s}^{+}\left(p^{k}\right) \backslash \Gamma_{n s}\left(p^{k}\right)$ we have:

$$
T_{h}^{+}(s(\tau))=-J_{s}(\tau) T_{h}^{+}(\tau) .
$$

Proof. It is clear from Proposition 4.1 that for every $s \in \Gamma_{n s}^{+}\left(p^{k}\right)$ there exists a $2 p^{k}$-th root of unity $c$ such that: $T_{h}^{+}(s(\tau))=c T_{h}^{+}(\tau) J_{s}(\tau)$ so it is natural to define:

$$
C_{h}(s)=\frac{T_{h}^{+}(s(\tau))}{T_{h}^{+}(\tau) J_{s}(\tau)} \in \boldsymbol{\mu}_{\mathbf{2} p^{k}}
$$

On the one hand:

$$
T_{h}^{+}\left(\left(s s^{\prime}\right)(\tau)\right)=C_{h}\left(s s^{\prime}\right) T_{h}^{+}(\tau) J_{s s^{\prime}}(\tau)
$$

on the other hand:

$T_{h}^{+}\left(s\left(s^{\prime}(\tau)\right)\right)=C_{h}(s) T_{h}^{+}\left(s^{\prime}(\tau)\right) J_{s}\left(s^{\prime}(\tau)\right)=C_{h}(s) C_{h}\left(s^{\prime}\right) J_{s}\left(s^{\prime}(\tau)\right) J_{s^{\prime}}(\tau) T_{h}^{+}(\tau)$.

Considering that $J_{s s^{\prime}}(\tau)=J_{s}\left(s^{\prime}(\tau)\right) J_{s^{\prime}}(\tau)$ we have:

$$
C_{h}\left(s s^{\prime}\right)=C_{h}(s) C_{h}\left(s^{\prime}\right) .
$$

From Proposition 6.3 we deduce easily that $C_{h}\left( \pm \Gamma\left(p^{k}\right)\right)=1$ for every $h$. So $C_{h}$ are characters of $\Gamma_{n s}^{+}\left(p^{k}\right) / \pm \Gamma\left(p^{k}\right)$. Since this quotient is isomorphic to $C_{n s}^{\prime+}\left(p^{k}\right) /\{ \pm I\}$ and since for every $\alpha \in C_{n s}^{\prime+}\left(p^{k}\right) \backslash C_{n s}^{\prime}\left(p^{k}\right)$ we have $\alpha^{2}=-I$, by Proposition 5.1 we obtain that $\Gamma_{n s}^{+}\left(p^{k}\right) / \pm \Gamma\left(p^{k}\right)$ is a dihedral group of $(p+1) p^{k-1}$ elements. These observations entail ipso facto that $C_{h}(s) \in\{ \pm 1\}$. As in Proposition 6.2 choose a matrix $\gamma_{r} \in S L_{2}(\mathbb{Z})$ lifting $M_{r} \in C_{n s}^{\prime}\left(p^{k}\right)$ where $r$ generates the subgroup of $\left(\mathbf{O}_{K} / p^{k} \mathbf{O}_{K}\right)^{*}$ of elements of norm 1. Choose $\gamma=\left(\begin{array}{ll}a & b \\ c & d\end{array}\right)$ in $\Gamma_{n s}^{+}\left(p^{k}\right) \backslash \Gamma_{n s}\left(p^{k}\right)$. It is not restrictive to suppose that $a=d \bmod 2$. If this did not happen we would alternatively choose:

$$
\gamma=\left(\begin{array}{ll}
a & b \\
c & d
\end{array}\right)\left(\begin{array}{cc}
1 & p^{k} \\
0 & 1
\end{array}\right)=\left(\begin{array}{ll}
a & a p^{k}+b \\
c & c p^{k}+d
\end{array}\right) .
$$


If $a \not \equiv d \bmod 2$ then $b$ and $c$ are inevitably odd because $a d-b c=1$ so the new coefficients on the diagonal verify $a \equiv c p^{k}+d \bmod 2$.

Notice that $\left\{\gamma \gamma_{r}^{j} \gamma^{-1}, \gamma \gamma_{r}^{j}\right\}_{j=1, \ldots, \frac{p+1}{2}\left(p^{k-1}\right)}$ is a set of representatives of cosets for the quotient group $\Gamma_{n s}^{+}\left(p^{k}\right) / \pm \Gamma\left(p^{k}\right)$. Furthermore if $h \in\left(\mathbb{Z} / p^{k} \mathbb{Z}\right)^{*} /\{ \pm 1\}$ and $s \in\left(\mathbf{O}_{K} / p^{k} \mathbf{O}_{K}\right)^{*} /\{ \pm 1\}$ with $\pm|s|=h$, there exists a $2 p^{k}$-th root of unity $c^{\prime}$ such that:

$$
T_{h}^{+}(\tau)=c^{\prime} \prod_{j=1}^{\frac{p+1}{2} p^{k-1}} \mathfrak{k}_{[s] \gamma \gamma_{r}^{j} \gamma^{-1}}(\tau) \prod_{j=1}^{\frac{p+1}{2} p^{k-1}} \mathfrak{k}_{[s] \gamma \gamma_{r}^{j}}(\tau)
$$

We calculate:

$$
\begin{aligned}
& T_{h}^{+}(\gamma(\tau))=c^{\prime} J_{\gamma}(\tau) \prod_{j=1}^{\frac{p+1}{2} p^{k-1}} \mathfrak{k}_{[s] \gamma \gamma_{r}^{j}}(\tau) \prod_{j=1}^{\frac{p+1}{2} p^{k-1}} \mathfrak{k}_{[s] \gamma \gamma_{r}^{j} \gamma}(\tau)= \\
& =c^{\prime}(-1)^{\frac{p+1}{2}} J_{\gamma}(\tau) \prod_{j=1}^{\frac{p+1}{2} p^{k-1}} \mathfrak{k}_{[s] \gamma \gamma_{r}^{j}}(\tau) \prod_{j=1}^{\frac{p+1}{2} p^{k-1}} \mathfrak{k}_{-[s] \gamma \gamma_{r}^{j} \gamma}(\tau) .
\end{aligned}
$$

But $\gamma \gamma_{r}^{j} \gamma^{-1} \equiv-\gamma \gamma_{r}^{j} \gamma \bmod p^{k}$ and $\gamma^{-1}+\gamma$ (in agreement with the previous convention) has all even coefficients so:

$$
[s] \gamma \gamma_{r}^{j} \gamma^{-1}-\left(-[s] \gamma \gamma_{r}^{j} \gamma\right)=[s] \gamma \gamma_{r}^{j}\left(\gamma^{-1}+\gamma\right) \in(2 \mathbb{Z})^{2}
$$

and considering Proposition 4.1 part (2) we have:

$$
\frac{\mathfrak{k}_{-[s] \gamma \gamma_{r}^{j} \gamma}(\tau)}{\mathfrak{k}_{[s] \gamma \gamma_{r}^{j} \gamma^{-1}}(\tau)} \in \boldsymbol{\mu}_{\boldsymbol{p}^{k}}
$$

Therefore:

$$
C_{h}(\gamma)=(-1)^{\frac{p+1}{2}} \prod_{j=1}^{\frac{p+1}{2} p^{k-1}} \frac{\mathfrak{k}_{-[s] \gamma \gamma_{r}^{j} \gamma}(\tau)}{\mathfrak{k}_{[s] \gamma \gamma_{r}^{j} \gamma^{-1}}(\tau)}
$$

so $C_{h}(\gamma)(-1)^{\frac{p+1}{2}} \in \boldsymbol{\mu}_{p^{k}} \cap\{ \pm 1\}$, we have necessarily $C_{h}(\gamma)=(-1)^{\frac{p+1}{2}}$ for every $\gamma \in \Gamma_{n s}^{+}\left(p^{k}\right) \backslash \Gamma_{n s}\left(p^{k}\right)$ and the proposition follows.

Theorem 6.5. If $p \neq 2,3$ the subgroup of modular units in $F_{p^{k}}$ of $X_{n s}^{+}\left(p^{k}\right)$ consists (modulo constants) of power products:

$$
g(\tau)=\prod_{h \in\left(\left(\mathbb{Z} / p^{k} \mathbb{Z}\right)^{*} /\{ \pm 1\}\right)} G_{h}^{+n_{h}^{+}}(\tau)
$$

where $d=\frac{12}{\operatorname{gcd}(12, p+1)}$ divides $\sum_{h} n_{h}^{+}$. 
Proof. By Proposition 6.2 and Theorem 4.3, every modular unit on $X_{n s}^{+}\left(p^{k}\right)$ can be written in the above indicated way. In fact, $d \mid \sum_{h} n_{h}^{+}$is equivalent to saying: $12 \mid(p+1) p^{k-1} \sum_{h} n_{h}^{+}$.

By Proposition 6.4 all the functions of this form are modular units of $X_{n s}^{+}\left(p^{k}\right)$. In fact, if $p \equiv 3 \bmod 4$, the functions $T_{h}^{+}(\tau)$ are nearly holomorphic modular forms for $X_{n s}^{+}\left(p^{k}\right)$. If $p \equiv 1 \bmod 4$, even if the functions $T_{h}^{+}(\tau)$ are not nearly holomorphic modular forms for $X_{n s}^{+}\left(p^{k}\right)$, the product $\prod_{h} T_{h}^{+n_{h}^{+}}(\tau)$ has this property, because $\sum_{h} n_{h}^{+}$is even in this case.

Notice that such a writing for $g(\tau)$ is not unique because of the fact that the following product is constant:

$$
\prod_{h \in\left(\left(\mathbb{Z} / p^{k} \mathbb{Z}\right)^{*} /\{ \pm 1\}\right)} G_{h}^{+}(\tau)=\prod_{t \in\left(\mathbf{O}_{K} / p^{k} \mathbf{O}_{K}\right)^{*} /\{ \pm 1\}} g_{[t]}(\tau) .
$$

Remark 6.6. Let $g$ be a generator of $\left(\mathbb{Z} / p^{k} \mathbb{Z}\right)^{*}$. Choose $s \in\left(\mathbf{O}_{K} / p^{k} \mathbf{O}_{K}\right)^{*}$ with $|s|=g$ and denote with $\rho \in \operatorname{Gal}\left(F_{p^{k}}, \mathbb{Q}(j)\right)$ the automorphism corresponding to the matrix $M_{s}$ respect to the isomorphism $\operatorname{Gal}\left(F_{p^{k}}, \mathbb{Q}(j)\right) \cong$ $G L_{2}\left(\mathbb{Z} / p^{k} \mathbb{Z}\right) / \pm I$ described in Theorem 2.2 , Let $F_{n s}^{+}\left(p^{k}\right)$ be the subfield of $F_{p^{k}}$ fixed by $C_{n s}^{\prime+}\left(p^{k}\right) / \pm I$. Choose $\sigma \in \operatorname{Gal}\left(F_{p^{k}}, \mathbb{Q}(j)\right)$. From Galois theory we have:

$$
\operatorname{Gal}\left(F_{p^{k}}, \sigma\left(F_{n s}^{+}\left(p^{k}\right)\right)\right)=\sigma \operatorname{Gal}\left(F_{p^{k}}, F_{n s}^{+}\left(p^{k}\right)\right) \sigma^{-1}
$$

thus saying that $\sigma\left(F_{n s}^{+}\left(p^{k}\right)\right)=F_{n s}^{+}\left(p^{k}\right)$ amounts to say that $\sigma$ belongs to the normalizer of $C_{n s}^{\prime+}\left(p^{k}\right) / \pm I$, in other words we have: $\sigma \in C_{n s}^{+}\left(p^{k}\right) / \pm I$. Consider $\sigma_{1}, \sigma_{2} \in C_{n s}^{+}\left(p^{k}\right) / \pm I$. We have $\sigma_{1}(f(\tau))=\sigma_{2}(f(\tau))$ for every $f(\tau) \in F_{n s}^{+}\left(p^{k}\right)$ if and only if $\sigma_{1} \sigma_{2}^{-1} \in C_{n s}^{\prime+}\left(p^{k}\right) / \pm I$ or equivalently $\operatorname{det} \sigma_{1}=$ $\operatorname{det} \sigma_{2}$. So every automorphism $\sigma \uparrow_{F_{n s}^{+}\left(p^{k}\right)}: F_{n s}^{+}\left(p^{k}\right) \rightarrow F_{n s}^{+}\left(p^{k}\right)$ fixing $\mathbb{Q}(j)$ can be written in the form $\sigma=\rho^{j}$ for some $0 \leq j \leq \varphi\left(p^{k}\right)-1$. Notice that if

$$
f(\tau)=\prod_{h \in\left(\left(\mathbb{Z} / p^{k} \mathbb{Z}\right)^{*} /\{ \pm 1\}\right)} G_{h}^{+n_{h}^{+}}(\tau)
$$

and

$$
h(\tau)=\prod_{h \in\left(\left(\mathbb{Z} / p^{k} \mathbb{Z}\right)^{*} /\{ \pm 1\}\right)} G_{h( \pm g)}^{+} n_{h}^{+}(\tau)
$$

are modular units on $X_{n s}^{+}\left(p^{k}\right)$, from proposition 4.2 we have:

$$
\begin{gathered}
(\rho(f(\tau)))^{12 p^{k}}=\rho\left(f(\tau)^{12 p^{k}}\right)=\rho\left(\prod_{h \in\left(\left(\mathbb{Z} / p^{k} \mathbb{Z}\right)^{*} /\{ \pm 1\}\right)} G_{h}^{+12 p^{k} n_{h}^{+}}(\tau)\right)= \\
=\prod_{h \in\left(\left(\mathbb{Z} / p^{k} \mathbb{Z}\right)^{*} /\{ \pm 1\}\right)} G_{h( \pm g)}^{+} 12 p^{k} n_{h}^{+}(\tau)=(h(\tau))^{12 p^{k}} .
\end{gathered}
$$


So $\rho(f(\tau))=\operatorname{ch}(\tau)$ for some $c \in \mathbb{Q}\left(\zeta_{p^{k}}\right)$ and all the functions $\rho^{j}(f(\tau))$ are modular units. Choosing $j=\frac{1}{2} \varphi\left(p^{k}\right)$ we deduce that for every modular unit $f(\tau)$ on $X_{n s}^{+}\left(p^{k}\right)$ there exist $c^{\prime} \in \mathbb{Q}\left(\zeta_{p^{k}}\right)$ such that:

$$
c^{\prime} f(\tau) \in \mathbb{Q}\left(\cos \left(\frac{2 \pi}{p^{k}}\right)\right)\left(\left(q^{p^{-k}}\right)\right) \text { with } q=e^{2 \pi i \tau} .
$$

\section{Cuspidal Divisor Class Group of non-split Cartan curves}

Let $p \geq 5$ a prime and let $R=\mathbb{Z}[H]$ be the group ring of $H=\left(\mathbb{Z} / p^{k} \mathbb{Z}\right)^{*} /\{ \pm 1\}$ over $\mathbb{Z}$. Let $w$ be a generator of $H$. For $\alpha \in \mathbb{Z} / p^{k} \mathbb{Z}$, let be $a \in \mathbb{Z}$ congruent to $\alpha \bmod p^{k}$. We define:

$$
\left\langle\frac{\alpha}{p}\right\rangle:=\left\langle\frac{a}{p}\right\rangle
$$

Define the Stickelberger element:

$$
\theta=\frac{p^{k}}{2} \sum_{i=1}^{\frac{p-1}{2} p^{k-1}} \sum_{\substack{\left(\left(\mathbf{O}_{K} / p^{k} \mathbf{O}_{K}\right)^{*} /\{ \pm 1\}\right) \\ \pm \mid=w^{i}}} B_{2}\left(\left\langle\frac{\frac{1}{2}(s+\bar{s})}{p^{k}}\right\rangle\right) w^{-i} \in \mathbb{Q}[H] .
$$

Define the ideals:

$$
\begin{gathered}
R_{0}:=\left\{\sum b_{j} w^{j} \in R \text { such that } \operatorname{deg}\left(\sum b_{j} w^{j}\right)=\sum b_{j}=0\right\}, \\
R_{d}:=\left\{\sum b_{j} w^{j} \in R \text { such that } d \text { divides } \operatorname{deg}\left(\sum b_{j} w^{j}\right)=\sum b_{j}\right\} .
\end{gathered}
$$

Now we can state the main result:

Main Theorem 7.1. The group generated by the divisors of modular units in $F_{p^{k}}$ of the curve $X_{n s}^{+}\left(p^{k}\right)$ can be expressed both as $R_{d} \theta$ and as Stickelberger module $R \theta \cap R$. The Cuspidal Divisor Class Group on $X_{n s}^{+}\left(p^{k}\right)$ is a module over $Z[H]$ and we have the following isomorphism:

$$
\mathfrak{C}_{n s}^{+}\left(p^{k}\right) \cong R_{0} / R_{d} \theta
$$

Proof. For every $i \in \mathbb{Z} / \frac{\varphi\left(p^{k}\right)}{2} \mathbb{Z}$ define:

$$
a_{i}=\frac{p^{k}}{2} \sum_{\substack{s \in\left(\left(\mathbf{O}_{K} / p^{k} \mathbf{O}_{K}\right)^{*} /\{ \pm 1\}\right) \\ \pm \mid=w^{i}}} B_{2}\left(\left\langle\frac{\frac{1}{2}(s+\bar{s})}{p^{k}}\right\rangle\right) .
$$


We identify the cusps of $X_{n s}^{+}\left(p^{k}\right)$ with the elements in $H=\left(\mathbb{Z} / p^{k} \mathbb{Z}\right)^{*} /\{ \pm 1\}$ as explained in Proposition 5.2. In consideration of Proposition 4.5 we obtain:

$$
\operatorname{div} G_{w^{j}}^{+d}(\tau)=d \sum_{i=1}^{\frac{p-1}{2} p^{k-1}} a_{i} w^{j-i}
$$

If $p \not \equiv 11 \bmod 12$, the function $G_{w^{j}}^{+}(\tau)$ is not $\Gamma_{n s}^{+}\left(p^{k}\right)$-invariant but with a slight abuse of notation we write:

$$
\operatorname{div} G_{w^{j}}^{+}(\tau)=\sum_{i=1}^{\frac{p-1}{2} p^{k-1}} a_{i} w^{j-i} .
$$

It is clear that $\operatorname{div} G_{w^{i}}^{+}(\tau) \in \mathbb{Q}[H]$ and $d \operatorname{div} G_{w^{i}}^{+}(\tau) \in R$. Consider the Stickelberger element:

$$
\begin{gathered}
\operatorname{div} G_{\{ \pm 1\}}^{+}(\tau)=\sum_{i=1}^{\frac{p-1}{2} p^{k-1}} a_{i} w^{-i}= \\
=\frac{p^{k}}{2} \sum_{i=1}^{\frac{p-1}{2} p^{k-1}} \sum_{ \pm|s|=w^{i}} B_{2}\left(\left\langle\frac{\frac{1}{2}(s+\bar{s})}{p^{k}}\right\rangle\right) w^{-i}=\theta .
\end{gathered}
$$

Notice that: $\operatorname{div} G_{w^{j}}^{+}(\tau)=w^{j} \theta$. By Theorem 6.5, a $\Gamma_{n s}^{+}\left(p^{k}\right)$-invariant function $g(\tau) \in F_{p^{k}}$ is a modular unit of $X_{n s}^{+}\left(p^{k}\right)$, if and only if $\operatorname{div} g(\tau) \in R_{d} \theta$. By [7, Proposition 2.3, Chapter 5] we have $R_{d} \theta=R \theta \cap R$.

Remark 7.2. Following Remark [6.6, consider $G:=\frac{C_{n s}^{+}\left(p^{k}\right) / \pm I}{C_{n s}^{\prime+}\left(p^{k}\right) / \pm I} \cong\left(\mathbb{Z} / p^{k} \mathbb{Z}\right)^{*}$ and let $\rho$ be a generator of $G /\{ \pm 1\}$ with $\pm \operatorname{det} \rho=w$. We may identify the group $H$ parameterizing the cusps of $X_{n s}^{+}\left(p^{k}\right)$ with $G /\{ \pm 1\}$ observing that for every automorphism $\rho^{j} \in G /\{ \pm 1\}$ and each moduar unit $h(\tau) \in F_{n s}^{+}\left(p^{k}\right)$ we have:

$$
\operatorname{ord}_{w^{-j}}(h(\tau))=\operatorname{ord}_{\rho^{-j}(\infty)}(h(\tau))=\operatorname{ord}_{\infty} \rho^{j}(h(\tau))
$$

and

$$
\operatorname{div}\left(\rho^{j}(h(\tau))\right)=w^{j} \operatorname{div}(h(\tau)) .
$$

If $\sum a_{j} \rho^{j} \in \mathbb{Z}[G /\{ \pm 1\}] \cong \mathbb{Z}[H]$ we define

$$
\left(\sum a_{j} w^{j}\right)(h(\tau))=\prod \rho^{j}(h(\tau))^{a_{j}}
$$

and clearly we have:

$$
\operatorname{div}\left(\prod \rho^{j}(h(\tau))^{a_{j}}\right)=\left(\sum a_{j} w^{j}\right) \operatorname{div}(h(\tau))
$$


so $\mathfrak{C}_{n s}^{+}\left(p^{k}\right)$ has a natural structure of $\mathbb{Z}[H]$-module which emphasizes the analogy with the classical theory of cyclotomic fields recalled in the introductory section.

Define:

$$
\theta^{\prime}=\theta-\frac{(p+1) p^{2 k-1}}{12} \sum_{i=1}^{\frac{p-1}{2} p^{k-1}} w^{i}
$$

and observe that $\theta^{\prime} \in R$ and $\operatorname{deg}\left(\theta^{\prime}\right)=-\frac{p^{2}-1}{24} p^{3 k-2}$.

Proposition 7.3. We have:

$$
R_{0} \cap\left(R \theta^{\prime}+p^{2 k-1} R \sum_{i=1}^{\frac{p-1}{2} p^{k-1}} w^{i}\right)=R_{d} \theta
$$

Proof. Let $\alpha, \beta \in R$ such that:

$$
\alpha \theta^{\prime}+p^{2 k-1} \beta \sum_{i=1}^{\frac{p-1}{2} p^{k-1}} w^{i} \in R_{0}
$$

Then

$$
-\operatorname{deg}(\alpha) \frac{p^{2}-1}{24} p^{3 k-2}+p^{2 k-1} \operatorname{deg}(\beta) \frac{p-1}{2} p^{k-1}=0
$$

implies $(p+1) \operatorname{deg}(\alpha)=12 \operatorname{deg}(\beta)$. This is equivalent to say:

$$
d=\frac{12}{\operatorname{gcd}(12, p+1)} \text { divides } \operatorname{deg}(\alpha)
$$

and

$$
\alpha \theta^{\prime}+p^{2 k-1} \beta \sum w^{i}=\alpha \theta^{\prime}+\frac{(p+1) p^{2 k-1} \operatorname{deg}(\alpha)}{12} \sum w^{i}=\alpha \theta
$$

Theorem 7.4. We have:

$$
\left|\mathfrak{C}_{n s}^{+}\left(p^{k}\right)\right|=\frac{\left|\operatorname{det} A_{\theta^{\prime}}\right|}{\frac{p^{2}-1}{24} p^{k-1} e}=24 \frac{\prod \frac{p^{k}}{2} B_{2, \chi}}{\operatorname{gcd}(12, p+1)(p-1) p^{k-1}},
$$

where $A_{\theta^{\prime}}$ is a circulant Toeplitz matrix, $e=p^{3 k-2} \frac{p-1}{2 d}$ and the product runs over all nontrivial characters $\chi$ of $C\left(p^{k}\right) / \pm I$ such that $\chi(M)=1$ for every $M \in C\left(p^{k}\right)$ with $\operatorname{det} M= \pm 1$. 
Proof. From Proposition 7.3 and the following isomorphism:

$$
\begin{gathered}
R_{0} /\left(R_{0} \cap\left(R \theta^{\prime}+p^{2 k-1} R \sum w^{i}\right)\right) \cong \\
\cong\left(R_{0}+R \theta^{\prime}+p^{2 k-1} R \sum w^{i}\right) /\left(R \theta^{\prime}+p^{2 k-1} R \sum w^{i}\right)
\end{gathered}
$$

we deduce that

$$
\left|\mathfrak{C}_{n s}^{+}\left(p^{k}\right)\right|=\left(R_{0}+R \theta^{\prime}+p^{2 k-1} R \sum w^{i}\right):\left(R \theta^{\prime}+p^{2 k-1} R \sum w^{i}\right) .
$$

From the following chain of consecutive inclusions:

$$
R \supset R_{0}+R \theta^{\prime}+p^{2 k-1} R \sum w^{i} \supset R \theta^{\prime}+p^{2 k-1} R \sum w^{i} \supset R \theta^{\prime}
$$

we obtain

$$
\left|\mathfrak{C}_{n s}^{+}\left(p^{k}\right)\right|=\frac{\left(R: R \theta^{\prime}\right)}{\left(R:\left(R_{0}+R \theta^{\prime}+p^{2 k-1} R \sum w^{i}\right)\right)\left(\left(R \theta^{\prime}+p^{2 k-1} R \sum w^{i}\right): R \theta^{\prime}\right)} .
$$

Define

$$
\begin{gathered}
e:=\operatorname{gcd}\left(\operatorname{deg}\left(\theta^{\prime}\right), p^{2 k-1} \operatorname{deg}\left(\sum w^{i}\right)\right)= \\
=p^{3 k-2} \operatorname{gcd}\left(\frac{p^{2}-1}{24}, \frac{p-1}{2}\right)=p^{3 k-2} \frac{p-1}{2 d} .
\end{gathered}
$$

It is clear that

$$
R_{0}+R \theta^{\prime}+p^{2 k-1} R \sum w^{i}=R_{e},
$$

where by $R_{e}$ we mean the ideal of $R$ consisting of elements whose degree is divisibile by $e$. So

$$
\left(R:\left(R_{0}+R \theta^{\prime}+p^{2 k-1} R \sum w^{i}\right)\right)=e .
$$

Regarding $\left(\left(R \theta^{\prime}+p^{2 k-1} R \sum w^{i}\right): R \theta^{\prime}\right)$, we observe that

$$
\left(R \theta^{\prime}+p^{2 k-1} R \sum w^{i}\right) / R \theta^{\prime} \cong\left(p^{2 k-1} R \sum w^{i}\right) /\left(p^{2 k-1} R \sum w^{i} \cap R \theta^{\prime}\right) .
$$

But $\prod_{h} G_{h}^{+n_{h}^{+}}$is constant if and only if all $n_{h}^{+}$are the same and so

$$
\operatorname{div} \prod_{h} G_{h}^{+n_{h}^{+}}=\left(\sum n_{h}^{+} h\right) \theta=\sum n_{h}^{+} h\left(\theta^{\prime}+\frac{(p+1) p^{2 k-1}}{12} \sum_{i=1}^{\frac{p-1}{2} p^{k-1}} w^{i}\right)=0
$$

implies

$$
\left(\sum n_{h}^{+} h\right) \theta^{\prime}=-\frac{(p+1) p^{2 k-1}}{12} \sum n_{h}^{+} \sum w^{i} \Longleftrightarrow n_{w}^{+}=n_{w^{2}}^{+}=n_{w^{3}}^{+}=\ldots=n_{ \pm 1}^{+} .
$$


But

$$
\left(\sum w^{i}\right) \theta^{\prime}=\operatorname{deg}\left(\theta^{\prime}\right) \sum w^{i}=-\frac{p^{2}-1}{24} p^{3 k-2} \sum w^{i}
$$

So:

$$
\left(R \theta^{\prime}+p^{2 k-1} R \sum w^{i}\right): R \theta^{\prime}=\frac{p^{2}-1}{24} p^{k-1}
$$

The last index we need to compute is $\left(R: R \theta^{\prime}\right)$. Write $\theta^{\prime}=\sum a_{i}^{\prime} w^{-i}$ and $a_{i}^{\prime}=a_{i}-\frac{p+1}{12} p^{2 k-1}$. Define the following matrix:

$$
A_{\theta^{\prime}}=\left(\begin{array}{cccccc}
a_{0}^{\prime} & a_{1}^{\prime} & a_{2}^{\prime} & \ldots & a_{\frac{p-1}{2} p^{k-1}-2}^{\prime} & a_{\frac{p-1}{2} p^{k-1}-1}^{\prime} \\
a_{\frac{p-1}{2} p^{k-1}-1}^{\prime} & a_{0}^{\prime} & a_{1}^{\prime} & \ldots & a_{\frac{p-1}{2} p^{k-1}-3}^{\prime} & a_{\frac{p-1}{2} p^{k-1}-2}^{\prime} \\
a_{\frac{p-1}{2} p^{k-1}-2}^{\prime} & a_{\frac{p-1}{2} p^{k-1}-1}^{\prime} & a_{0}^{\prime} & \ldots & a_{\frac{p-1}{2} p^{k-1}-4}^{\prime} & a_{\frac{p-1}{2} p^{k-1}-3}^{\prime} \\
\ldots \ldots \ldots \ldots \ldots \ldots \ldots \ldots \ldots & \ldots \ldots \ldots \ldots \ldots & \ldots \ldots \\
a_{2}^{\prime} & a_{3}^{\prime} & a_{4}^{\prime} & \ldots & a_{0}^{\prime} & a_{1}^{\prime} \\
a_{1}^{\prime} & a_{2}^{\prime} & a_{3}^{\prime} & \ldots & a_{\frac{p-1}{2} p^{k-1}-1}^{\prime} & a_{0}^{\prime}
\end{array}\right) .
$$

We have: $\left(R: R \theta^{\prime}\right)=\left|\operatorname{det} A_{\theta^{\prime}}\right|$. The matrix $A_{\theta^{\prime}}$ is a circulant Toeplitz matrix, in other words the coefficients $\left(A_{\theta^{\prime}}\right)_{i, j}$ depend only on $i-j \bmod \frac{p-1}{2} p^{k-1}$. This is the matrix of multiplication by $\theta^{\prime}$ in $\mathbb{C}[H]$, so we easily deduce that for $n=1,2, \ldots, \frac{p-1}{2} p^{k-1}$ the eighenvalues of $A_{\theta^{\prime}}$ are:

$$
\lambda_{n}=\sum_{j=1}^{\frac{p-1}{2} p^{k-1}} a_{j}^{\prime} e^{\frac{4 \pi i j n}{(p-1) p^{k-1}}}
$$

with corresponding eighenvectors:

$$
v_{n}=\sum_{j=1}^{\frac{p-1}{2} p^{k-1}} e^{\frac{4 \pi i j n}{(p-1) p^{k-1}}} w^{j}
$$

Observe that $\lambda_{\frac{p-1}{2} p^{k-1}}=\sum a_{i}^{\prime}=\operatorname{deg}\left(\theta^{\prime}\right)=-\frac{p^{2}-1}{24} p^{3 k-2}$ and that according to the definition of Theorem 4.6 the others $\lambda_{n}$ correspond to the generalized Bernoulli number $\frac{p^{k}}{2} B_{2, \chi}$ where $\chi$ runs over the nontrivial characters of $C\left(p^{k}\right) / \pm I$ such that $\chi(M)=1$ for every $M \in C\left(p^{k}\right)$ with $\operatorname{det} M= \pm 1$.

Gathering all this information together we obtain the desired result.

\section{Explicit calculation}

In this section we examine the curve $X_{n s}^{+}(p)$ more in details. Denote with $v$ a generator of the multiplicative group of $\mathbb{F}_{p^{2}}$ and indicate with $\omega$ a generator 
of the character group $\mathbb{F}_{p^{2}}^{*}$ viewing $C(p) \cong \mathbb{F}_{p^{2}}^{*}$. By Theorem 7.4, in this case we have:

$$
\begin{gathered}
B_{2, \chi}=\sum_{x \in \mathbb{F}_{p^{2}} / \pm 1} B_{2}\left(\left\langle\frac{\frac{1}{2} \operatorname{Tr}(x)}{p}\right\rangle\right) \chi(x), \\
\left|\mathfrak{C}_{n s}^{+}(p)\right|=\frac{24}{(p-1) \operatorname{gcd}(12, p+1)} \prod_{j=1}^{\frac{p-3}{2}} \frac{p}{2} B_{2, \omega^{(2 p+2) j}}= \\
=\frac{576\left|\operatorname{det}\left[\frac{p}{2}\left(\sum_{l=0}^{p} B_{2}\left(\left\langle\frac{\frac{1}{2} \operatorname{Tr}\left(v^{i-j+l \frac{p-1}{2}}\right)}{p}\right\rangle\right)-\frac{p+1}{6}\right)\right]_{1 \leq i, j \leq \frac{p-1}{2}}\right|}{(p-1)^{2} p(p+1) \operatorname{gcd}(12, p+1)} .
\end{gathered}
$$

In the following table we show the factorization of the orders of cuspidal

\begin{tabular}{|c|c|}
\hline$p$ & $\left|\mathfrak{C}_{n s}^{+}(p)\right|$ \\
\hline 5 & 1 \\
\hline 7 & 1 \\
\hline 11 & 11 \\
\hline 13 & $7 \cdot 13^{2}$ \\
\hline 17 & $2^{4} \cdot 3 \cdot 17^{3}$ \\
\hline 19 & $3 \cdot 19^{3} \cdot 487$ \\
\hline 23 & $23^{4} \cdot 37181$ \\
\hline 29 & $2^{6} \cdot 5 \cdot 7^{2} \cdot 29^{6} \cdot 43^{2}$ \\
\hline 31 & $2^{2} \cdot 5 \cdot 7 \cdot 11 \cdot 31^{6} \cdot 2302381$ \\
\hline 37 & $3^{4} \cdot 7^{2} \cdot 19^{3} \cdot 37^{8} \cdot 577^{2}$ \\
\hline 41 & $2^{6} \cdot 5^{2} \cdot 7 \cdot 31^{4} \cdot 41^{9} \cdot 431^{2}$ \\
\hline 43 & $2^{2} \cdot 19 \cdot 29 \cdot 43^{9} \cdot 463 \cdot 1051 \cdot 416532733$ \\
\hline 53 & $3^{2} \cdot 13^{2} \cdot 53^{12} \cdot 96331^{2} \cdot 379549^{2}$ \\
\hline 59 & $59^{14} \cdot 9988553613691393812358794271$ \\
\hline 67 & $67^{16} \cdot 193 \cdot 661^{2} \cdot 2861 \cdot 8009 \cdot 11287 \cdot 9383200455691459$ \\
\hline 71 & $31 \cdot 71^{16} \cdot 113 \cdot 211 \cdot 281 \cdot 701^{2} \cdot 12713 \cdot 13070849919225655729061$ \\
\hline 73 & $2^{2} \cdot 3^{4} \cdot 11^{2} \cdot 37 \cdot 73^{17} \cdot 79^{2} \cdot 241^{2} \cdot 3341773^{2} \cdot 11596933^{2}$ \\
\hline 83 & $83^{19} \cdot 17210653 \cdot 151251379 \cdot 18934761332741 \cdot 48833370476331324749419$ \\
\hline 89 & $2^{2} \cdot 3 \cdot 5 \cdot 11^{2} \cdot 13^{2} \cdot 89^{21} \cdot 4027^{2} \cdot 262504573^{2} \cdot 15354699728897^{2}$ \\
\hline 101 & $5^{4} \cdot 17 \cdot 101^{24} \cdot 52951^{2} \cdot 54371^{2} \cdot 58884077243434864347851^{2}$ \\
\hline
\end{tabular}
divisor class groups $\mathfrak{C}_{n s}^{+}(p)$ for some primes $p \leq 101$ :

Table 8.1.

We recall the following result of [1]:

Theorem 8.2. The genera of $X_{n s}^{+}(p)$ are:

$$
g\left(X_{n s}^{+}(p)\right)=\frac{1}{24}\left(p^{2}-10 p+23+6\left(\frac{-1}{p}\right)+4\left(\frac{-3}{p}\right)\right) .
$$


Proof. It is a consequence of Hurwitz's formula [17, Proposition 1.40]:

$$
g(\Gamma)=1+\frac{d}{12}-\frac{e_{2}}{4}-\frac{e_{3}}{3}-\frac{e_{\infty}}{2} .
$$

In this case: $d:=\left[S L_{2}(\mathbb{Z}): \Gamma_{n s}^{+}(p)\right]=\frac{p(p-1)}{2}, e_{\infty}=\frac{p-1}{2}$ is the number of cusps (see Proposition 5.2), $e_{2}$ and $e_{3}$ denote the number of elliptic points of period 2 and 3. We have (cfr. [13, Proposition 12]):

$$
e_{2}=\frac{p+1}{2}-\left(\frac{-1}{p}\right) \text { and } e_{3}=\frac{1}{2}-\frac{1}{2}\left(\frac{-3}{p}\right) .
$$

By Theorem 8.2 we have $g\left(X_{n s}^{+}(5)\right)=g\left(X_{n s}^{+}(7)\right)=0$ so it will not be surprising to find out that $\mathfrak{C}_{n s}^{+}(5)$ and $\mathfrak{C}_{n s}^{+}(7)$ are trivial.

For $11 \leq p \leq 31$ we provide further corroborative evidence of Table 8.1 , From [15, p. 195] we have:

Theorem 8.3. The modular curve $X_{n s}^{+}(p)$ associated to the subgroup $C_{n s}^{+}(p)$ is a projective non-singular modular curve which can be defined over $\mathbb{Q}$. The cusps are defined over $\mathbb{Q}\left(\cos \left(\frac{2 \pi}{p}\right)\right)$, the maximal real subfield of the $p$-th cyclotomic field.

From [3] we have the following result:

Theorem 8.4. The jacobian of $X_{n s}^{+}(p)$ is isogenous to the new part of the Jacobian $J_{0}^{+}\left(p^{2}\right)$ of $X_{0}^{+}\left(p^{2}\right)$.

From [14, Chapter 12] we have this interesting corollary of the EichlerShimura relation [4, pag. 354]:

Theorem 8.5. Let $q$ be a prime that does not divide $N$ and let $f(x)$ the characteristic polynomial of the Hecke operator $T_{q}$ acting on $S_{2}^{\text {new }}\left(\Gamma_{0}^{+}(N)\right)$. Then:

$$
\left|J_{0}^{+ \text {new }}(N)\left(\mathbb{F}_{q}\right)\right|=f(q+1) .
$$

Choose a prime $q \equiv \pm 1 \bmod p$ that does not divide $\left|\mathfrak{C}_{n s}^{+}(p)\right|$. From the previous theorems, the cuspidal divisor class group $\mathfrak{C}_{n s}^{+}(p)$ injects into $J_{n s}^{+}(p)\left(\mathbb{F}_{q}\right)$. So we expect that $\left|\mathfrak{C}_{n s}^{+}(p)\right|$ divides $\left|J_{0}^{+ \text {new }}\left(p^{2}\right)\left(\mathbb{F}_{q}\right)\right|=f_{q, p^{2}}(q+1)$ where $f_{q, p^{2}}$ is the characteristic polynomial of the Hecke operator $T_{q}$ acting on $S_{2}^{\text {new }}\left(\Gamma_{0}^{+}\left(p^{2}\right)\right)$. From the modular form database of W.Stein we have:

$$
\begin{aligned}
& \left|J_{0}^{+ \text {new }}\left(11^{2}\right)\left(\mathbb{F}_{23}\right)\right|=f_{23,121}(24)=3 \cdot 11 \\
& \left|J_{0}^{+ \text {new }}\left(11^{2}\right)\left(\mathbb{F}_{43}\right)\right|=f_{43,121}(44)=2^{2} \cdot 11
\end{aligned}
$$




$$
\begin{aligned}
& \left|J_{0}^{+ \text {new }}\left(11^{2}\right)\left(\mathbb{F}_{67}\right)\right|=f_{67,121}(68)=5 \cdot 11 \text {, } \\
& \left|J_{0}^{+ \text {new }}\left(11^{2}\right)\left(\mathbb{F}_{89}\right)\right|=f_{89,121}(90)=3^{2} \cdot 11 \text {, } \\
& \left|J_{0}^{+ \text {new }}\left(11^{2}\right)\left(\mathbb{F}_{109}\right)\right|=f_{109,121}(110)=2 \cdot 5 \cdot 11 \text {, } \\
& \left|J_{0}^{+ \text {new }}\left(11^{2}\right)\left(\mathbb{F}_{131}\right)\right|=f_{131,121}(132)=2^{2} \cdot 3 \cdot 11 \text {, } \\
& \left|J_{0}^{+ \text {new }}\left(11^{2}\right)\left(\mathbb{F}_{197}\right)\right|=f_{197,121}(198)=2 \cdot 3^{2} \cdot 11 \text {, } \\
& \left|J_{0}^{+ \text {new }}\left(11^{2}\right)\left(\mathbb{F}_{199}\right)\right|=f_{199,121}(200)=2^{2} \cdot 5 \cdot 11 \text {, } \\
& \left|J_{0}^{+ \text {new }}\left(11^{2}\right)\left(\mathbb{F}_{241}\right)\right|=f_{241,121}(242)=2 \cdot 11^{2} \text {, } \\
& \left|J_{0}^{+ \text {new }}\left(11^{2}\right)\left(\mathbb{F}_{263}\right)\right|=f_{263,121}(264)=2^{3} \cdot 3 \cdot 11 \text {, } \\
& \left|J_{0}^{+ \text {new }}\left(11^{2}\right)\left(\mathbb{F}_{307}\right)\right|=f_{307,121}(308)=2^{2} \cdot 7 \cdot 11 \text {, } \\
& \left|J_{0}^{+ \text {new }}\left(11^{2}\right)\left(\mathbb{F}_{331}\right)\right|=f_{331,121}(332)=3^{3} \cdot 11 \text {, } \\
& \left|J_{0}^{+ \text {new }}\left(11^{2}\right)\left(\mathbb{F}_{353}\right)\right|=f_{353,121}(354)=3 \cdot 11^{2} \text {, } \\
& \left|J_{0}^{+ \text {new }}\left(11^{2}\right)\left(\mathbb{F}_{373}\right)\right|=f_{373,121}(374)=2 \cdot 11 \cdot 17 \text {, } \\
& \left|J_{0}^{+ \text {new }}\left(11^{2}\right)\left(\mathbb{F}_{397}\right)\right|=f_{397,121}(398)=2^{2} \cdot 3^{2} \cdot 11 \text {, } \\
& \left|J_{0}^{+ \text {new }}\left(11^{2}\right)\left(\mathbb{F}_{419}\right)\right|=f_{419,121}(420)=2^{2} \cdot 3^{2} \cdot 11 \text {, } \\
& \left|J_{0}^{+ \text {new }}\left(11^{2}\right)\left(\mathbb{F}_{439}\right)\right|=f_{439,121}(440)=2^{3} \cdot 5 \cdot 11 \text {, } \\
& \left|J_{0}^{+ \text {new }}\left(11^{2}\right)\left(\mathbb{F}_{461}\right)\right|=f_{461,121}(462)=2 \cdot 3 \cdot 7 \cdot 11 \text {, } \\
& \left|J_{0}^{+ \text {new }}\left(11^{2}\right)\left(\mathbb{F}_{463}\right)\right|=f_{463,121}(464)=3^{2} \cdot 5 \cdot 11 \text {, } \\
& \left|J_{0}^{+ \text {new }}\left(13^{2}\right)\left(\mathbb{F}_{53}\right)\right|=f_{53,169}(54)=7 \cdot 13^{2} \cdot 127 \text {, } \\
& \left|J_{0}^{+ \text {new }}\left(13^{2}\right)\left(\mathbb{F}_{79}\right)\right|=f_{79,169}(80)=7 \cdot 13^{2} \cdot 449 \text {, } \\
& \left|J_{0}^{+ \text {new }}\left(13^{2}\right)\left(\mathbb{F}_{103}\right)\right|=f_{103,169}(104)=7 \cdot 13^{2} \cdot 967 \text {, } \\
& \left|J_{0}^{+ \text {new }}\left(13^{2}\right)\left(\mathbb{F}_{131}\right)\right|=f_{131,169}(132)=7 \cdot 13^{5} \text {, } \\
& \left|J_{0}^{+ \text {new }}\left(13^{2}\right)\left(\mathbb{F}_{157}\right)\right|=f_{157,169}(158)=7^{2} \cdot 13^{2} \cdot 503 \text {, } \\
& \left|J_{0}^{+ \text {new }}\left(13^{2}\right)\left(\mathbb{F}_{181}\right)\right|=f_{181,169}(182)=7 \cdot 13^{2} \cdot 4327 \text {, } \\
& \left|J_{0}^{+ \text {new }}\left(13^{2}\right)\left(\mathbb{F}_{233}\right)\right|=f_{233,169}(234)=7 \cdot 13^{2} \cdot 11731 \text {, } \\
& \left|J_{0}^{+ \text {new }}\left(13^{2}\right)\left(\mathbb{F}_{311}\right)\right|=f_{311,169}(312)=7 \cdot 13^{2} \cdot 26249 \text {, } \\
& \left|J_{0}^{+ \text {new }}\left(13^{2}\right)\left(\mathbb{F}_{313}\right)\right|=f_{313,169}(314)=7 \cdot 13^{2} \cdot 29443 \text {, } \\
& \left|J_{0}^{+ \text {new }}\left(13^{2}\right)\left(\mathbb{F}_{337}\right)\right|=f_{337,169}(338)=7 \cdot 13^{2} \cdot 35449 \text {, } \\
& \left|J_{0}^{+ \text {new }}\left(13^{2}\right)\left(\mathbb{F}_{389}\right)\right|=f_{389,169}(390)=2^{3} \cdot 7 \cdot 13^{2} \cdot 71 \cdot 83 \text {, } \\
& \left|J_{0}^{+ \text {new }}\left(13^{2}\right)\left(\mathbb{F}_{443}\right)\right|=f_{443,169}(444)=2^{3} \cdot 7 \cdot 13^{3} \cdot 643 \text {, } \\
& \left|J_{0}^{+ \text {new }}\left(13^{2}\right)\left(\mathbb{F}_{467}\right)\right|=f_{467,169}(468)=7 \cdot 13^{2} \cdot 93199 \text {, } \\
& \left|J_{0}^{+ \text {new }}\left(17^{2}\right)\left(\mathbb{F}_{67}\right)\right|=f_{67,289}(68)=2^{8} \cdot 3 \cdot 17^{5} \cdot 71 \text {, } \\
& \left|J_{0}^{+ \text {new }}\left(17^{2}\right)\left(\mathbb{F}_{101}\right)\right|=f_{101,289}(102)=2^{4} \cdot 3^{2} \cdot 7 \cdot 17^{3} \cdot 19 \cdot 79 \cdot 181 \text {, } \\
& \left|J_{0}^{+ \text {new }}\left(17^{2}\right)\left(\mathbb{F}_{103}\right)\right|=f_{103,289}(104)=2^{7} \cdot 3^{4} \cdot 17^{4} \cdot 1601, \\
& \left|J_{0}^{+ \text {new }}\left(17^{2}\right)\left(\mathbb{F}_{137}\right)\right|=f_{137,289}(138)=2^{6} \cdot 3^{8} \cdot 17^{4} \cdot 181 \text {, } \\
& \left|J_{0}^{+ \text {new }}\left(17^{2}\right)\left(\mathbb{F}_{239}\right)\right|=f_{239,289}(240)=2^{8} \cdot 3^{2} \cdot 17^{3} \cdot 373 \cdot 48871 \text {, } \\
& \left|J_{0}^{+ \text {new }}\left(17^{2}\right)\left(\mathbb{F}_{271}\right)\right|=f_{271,289}(272)=2^{5} \cdot 3^{9} \cdot 5^{3} \cdot 17^{4} \cdot 53 \text {, }
\end{aligned}
$$


$\left|J_{0}^{+ \text {new }}\left(17^{2}\right)\left(\mathbb{F}_{307}\right)\right|=f_{307,289}(308)=2^{6} \cdot 3^{5} \cdot 5 \cdot 17^{3} \cdot 23 \cdot 71 \cdot 1423$,
$\left|J_{0}^{+ \text {new }}\left(17^{2}\right)\left(\mathbb{F}_{373}\right)\right|=f_{373,289}(374)=2^{4} \cdot 3^{4} \cdot 17^{3} \cdot 23 \cdot 73 \cdot 101 \cdot 2789$,
$\left|J_{0}^{+ \text {new }}\left(17^{2}\right)\left(\mathbb{F}_{409}\right)\right|=f_{409,289}(410)=2^{7} \cdot 3^{5} \cdot 17^{3} \cdot 23 \cdot 53 \cdot 71 \cdot 359$,
$\left|J_{0}^{+ \text {new }}\left(17^{2}\right)\left(\mathbb{F}_{443}\right)\right|=f_{443,289}(444)=2^{5} \cdot 3^{2} \cdot 13 \cdot 17^{4} \cdot 19 \cdot 79 \cdot 15263$,
$\left|J_{0}^{+ \text {new }}\left(19^{2}\right)\left(\mathbb{F}_{37}\right)\right|=f_{37,361}(38)=2 \cdot 3 \cdot 19^{3} \cdot 37 \cdot 487 \cdot 5441$,
$\left|J_{0}^{+ \text {new }}\left(19^{2}\right)\left(\mathbb{F}_{113}\right)\right|=f_{113,361}(114)=2^{5} \cdot 3^{7} \cdot 19^{7} \cdot 487$
$\left|J_{0}^{+ \text {new }}\left(19^{2}\right)\left(\mathbb{F}_{151}\right)\right|=f_{151,361}(152)=2^{3} \cdot 3^{3} \cdot 17 \cdot 19^{4} \cdot 487 \cdot 1459141$,
$\left|J_{0}^{+ \text {new }}\left(19^{2}\right)\left(\mathbb{F}_{191}\right)\right|=f_{191,361}(192)=3^{2} \cdot 11^{5} \cdot 19^{6} \cdot 73 \cdot 487$
$\left|J_{0}^{+ \text {new }}\left(19^{2}\right)\left(\mathbb{F}_{227}\right)\right|=f_{227,361}(228)=2^{2} \cdot 3^{4} \cdot 19^{3} \cdot 487 \cdot 971 \cdot 7323581$,
$\left|J_{0}^{+ \text {new }}\left(19^{2}\right)\left(\mathbb{F}_{229}\right)\right|=f_{229,361}(230)=3 \cdot 11 \cdot 17 \cdot 19^{3} \cdot 467 \cdot 487 \cdot 2819^{2}$,
$\left|J_{0}^{+ \text {new }}\left(19^{2}\right)\left(\mathbb{F}_{379}\right)\right|=f_{379,361}(380)=2^{6} \cdot 3 \cdot 5^{2} \cdot 19^{3} \cdot 179 \cdot 487 \cdot 4019 \cdot 33247$,
$\left|J_{0}^{+ \text {new }}\left(19^{2}\right)\left(\mathbb{F}_{419}\right)\right|=f_{419,361}(420)=2^{6} \cdot 3^{2} \cdot 5^{3} \cdot 19^{3} \cdot 487 \cdot 509^{2} \cdot 16487$,
$\left|J_{0}^{+ \text {new }}\left(19^{2}\right)\left(\mathbb{F}_{457}\right)\right|=f_{457,361}(458)=2^{4} \cdot 3 \cdot 5^{4} \cdot 19^{3} \cdot 487 \cdot 521^{2} \cdot 65629$,
$\left|J_{0}^{+ \text {new }}\left(23^{2}\right)\left(\mathbb{F}_{47}\right)\right|=f_{47,529}(48)=2^{3} \cdot 3^{3} \cdot 7^{4} \cdot 11 \cdot 13 \cdot 23^{4} \cdot 8117 \cdot 37181$,
$\left|J_{0}^{+ \text {new }}\left(23^{2}\right)\left(\mathbb{F}_{137}\right)\right|=f_{137,529}(138)=2^{4} \cdot 3^{6} \cdot 23^{8} \cdot 2399 \cdot 37181 \cdot 75553$,
$\left|J_{0}^{+ \text {new }}\left(23^{2}\right)\left(\mathbb{F}_{139}\right)\right|=f_{139,529}(140)=2^{4} \cdot 3^{8} \cdot 23^{9} \cdot 107^{2} \cdot 109 \cdot 37181$,
$\left|J_{0}^{+ \text {new }}\left(23^{2}\right)\left(\mathbb{F}_{229}\right)\right|=f_{229,529}(230)=2^{6} \cdot 11 \cdot 23^{6} \cdot 43 \cdot 67 \cdot 37181 \cdot 325729 \cdot 1296721$,
$\left|J_{0}^{+ \text {new }}\left(23^{2}\right)\left(\mathbb{F}_{277}\right)\right|=f_{277,529}(278)=2^{8} \cdot 3^{10} \cdot 23^{7} \cdot 113^{2} \cdot 331 \cdot 7193 \cdot 37181$,
$\left|J_{0}^{+ \text {new }}\left(23^{2}\right)\left(\mathbb{F}_{367}\right)\right|=f_{367,529}(368)=2^{4} \cdot 23^{5} \cdot 67^{2} \cdot 193 \cdot 1847 \cdot 37181 \cdot 44617 \cdot$ 8643209 ,

$\left|J_{0}^{+ \text {new }}\left(23^{2}\right)\left(\mathbb{F}_{461}\right)\right|=f_{461,529}(462)=3^{6} \cdot 7^{4} \cdot 23^{7} \cdot 43^{2} \cdot 67 \cdot 199 \cdot 2857^{2} \cdot 37181$,

$\left|J_{0}^{+ \text {new }}\left(29^{2}\right)\left(\mathbb{F}_{59}\right)\right|=f_{59,841}(60)=2^{8} \cdot 3^{2} \cdot 5 \cdot 7^{2} \cdot 11^{2} \cdot 17 \cdot 23^{2} \cdot 29^{6} \cdot 43^{2} \cdot 569$. $967^{2} \cdot 2999 \cdot 11695231$ $\left|J_{0}^{+ \text {new }}\left(29^{2}\right)\left(\mathbb{F}_{173}\right)\right|=f_{173,841}(174)=2^{10} \cdot 3^{2} \cdot 5^{2} \cdot 7^{2} \cdot 29^{6} \cdot 31 \cdot 41^{2} \cdot 43^{2} \cdot 89$. $419^{2} \cdot 719 \cdot 1061 \cdot 36571 \cdot 1269691 \cdot 1909421$, $\left|J_{0}^{+ \text {new }}\left(29^{2}\right)\left(\mathbb{F}_{233}\right)\right|=f_{233,841}(234)=2^{10} \cdot 3^{2} \cdot 5 \cdot 7^{2} \cdot 29^{6} \cdot 43^{2} \cdot 167^{2} \cdot 211^{2} \cdot 421$. $1049 \cdot 3989 \cdot 317321 \cdot 422079165281099$, $\left|J_{0}^{+ \text {new }}\left(29^{2}\right)\left(\mathbb{F}_{347}\right)\right|=f_{347,841}(348)=2^{8} \cdot 3^{12} \cdot 5^{3} \cdot 7^{2} \cdot 11 \cdot 23^{2} \cdot 29^{6} \cdot 31 \cdot 43^{2}$. $71 \cdot 127^{2} \cdot 967^{2} \cdot 9601 \cdot 783719 \cdot 7292986801$ $\left|J_{0}^{+ \text {new }}\left(29^{2}\right)\left(\mathbb{F}_{349}\right)\right|=f_{349,841}(350)=2^{8} \cdot 5^{9} \cdot 7^{2} \cdot 13^{2} \cdot 19 \cdot 23 \cdot 29^{7} \cdot 43^{2} \cdot 83^{2}$. $103 \cdot 211 \cdot 3786151 \cdot 92610181 \cdot 3477902249$, $\left|J_{0}^{+ \text {new }}\left(29^{2}\right)\left(\mathbb{F}_{463}\right)\right|=f_{463,841}(464)=2^{13} \cdot 5^{7} \cdot 7^{7} \cdot 29^{6} \cdot 43^{3} \cdot 59 \cdot 97^{3} \cdot 461^{3}$. $1459 \cdot 23656223369 \cdot 230667656992649$,

$\left|J_{0}^{+ \text {new }}\left(31^{2}\right)\left(\mathbb{F}_{61}\right)\right|=f_{61,961}(62)=2^{10} \cdot 5 \cdot 7 \cdot 11 \cdot 31^{7} \cdot 137 \cdot 179 \cdot 1249 \cdot 10369$. 
$26699 \cdot 38177 \cdot 2302381 \cdot 24080801$,

$\left|J_{0}^{+ \text {new }}\left(31^{2}\right)\left(\mathbb{F}_{311}\right)\right|=f_{311,961}(312)=2^{8} \cdot 3^{2} \cdot 5 \cdot 7^{2} \cdot 11 \cdot 31^{7} \cdot 409 \cdot 3793^{2} \cdot 51551^{2}$.

$162691 \cdot 2302381 \cdot 22340831^{2} \cdot 24037019$,

$\left|J_{0}^{+ \text {new }}\left(31^{2}\right)\left(\mathbb{F}_{373}\right)\right|=f_{373,961}(374)=2^{4} \cdot 5 \cdot 7^{2} \cdot 11^{2} \cdot 13^{2} \cdot 31^{6} \cdot 251 \cdot 449$.

$2302381 \cdot 366424077359 \cdot 13600706515978033^{2}$,

$\left|J_{0}^{+ \text {new }}\left(31^{2}\right)\left(\mathbb{F}_{433}\right)\right|=f_{433,961}(434)=2^{6} \cdot 3^{6} \cdot 5 \cdot 7 \cdot 11 \cdot 17 \cdot 31^{11} \cdot 89 \cdot 97 \cdot 191$.

$401 \cdot 1153 \cdot 54331 \cdot 126961 \cdot 2302381 \cdot 12958271 \cdot 53053053405791$.

For $11 \leq p \leq 23$ we have:

$$
\underset{\substack{q<500 \text { prime, } \\ q \equiv \pm 1 \text { mod } p}}{\operatorname{gcd}}\left|J_{0}^{+ \text {new }}\left(p^{2}\right)\left(\mathbb{F}_{q}\right)\right|=\left|\mathfrak{C}_{n s}^{+}(p)\right|
$$

For $p=29$ and $p=31$ we have:

$$
\underset{\substack{q<500 \text { prime, } \\ q \equiv \pm 1 \bmod p}}{\operatorname{gcd}}\left|J_{0}^{+ \text {new }}\left(p^{2}\right)\left(\mathbb{F}_{q}\right)\right|=4\left|\mathfrak{C}_{n s}^{+}(p)\right| .
$$

We can improve the result by using the isogeny (cfr. [4, Paragraph 6.6]):

$$
J_{0}^{+n e w}\left(p^{2}\right) \longrightarrow \bigoplus_{f} A_{p, f}^{\prime}
$$

where the sum is taken over the equivalence classes of newforms $f \in S_{2}\left(\Gamma_{0}^{+}\left(p^{2}\right)\right)$.

Two forms $f$ and $g$ are declared equivalent if $g=f^{\sigma}$ for some automorphism $\sigma: \mathbb{C} \longrightarrow \mathbb{C}$. Denote with $\mathbb{K}_{f}$ the number field of $f$. We have:

$$
\begin{aligned}
& \underset{\substack{q<500 \text { prime, } \\
q \equiv \pm 1 \text { mod } 29}}{\operatorname{gcd}}\left|A_{29, f_{1}}^{\prime}\left(\mathbb{F}_{q}\right)\right|=7^{2} \text { where } \mathbb{K}_{f_{1}}=\mathbb{Q}(\sqrt{2}), \\
& \underset{\substack{q<500 \text { prime, } \\
q \equiv \pm 1 \text { mod } 29}}{\text { gcd }}\left|A_{29, f_{2}}^{\prime}\left(\mathbb{F}_{q}\right)\right|=29 \text { where } \mathbb{K}_{f_{2}}=\mathbb{Q}(\sqrt{5}), \\
& \underset{\substack{q<50 \text { prime, } \\
q \equiv \pm 1 \text { mod } 29}}{\text { gcd }}\left|A_{29, f_{3}}^{\prime}\left(\mathbb{F}_{q}\right)\right|=\underset{\substack{q<500 \text { prime, } \\
q \equiv \pm 1 \bmod 29}}{\text { gcd }}\left|A_{29, f_{4}}^{\prime}\left(\mathbb{F}_{q}\right)\right|=2^{3} \cdot 43
\end{aligned}
$$

where $\mathbb{K}_{f_{3}}=\mathbb{K}_{f_{4}}$ and $\left[\mathbb{K}_{f_{3}}: \mathbb{Q}\right]=3$,

$$
\begin{aligned}
& \underset{\substack{q<500 \text { prime, } \\
q \equiv \pm 1 \text { mod } 29}}{\operatorname{gcd}}\left|A_{29, f_{5}}^{\prime}\left(\mathbb{F}_{q}\right)\right|=5 \cdot 29^{2} \text { where }\left[\mathbb{K}_{f_{5}}: \mathbb{Q}\right]=6, \\
& \underset{\substack{q<500 \text { prime, } \\
q \equiv \pm 1 \text { mod } 29}}{\text { gcd }}\left|A_{29, f_{6}}^{\prime}\left(\mathbb{F}_{q}\right)\right|=29^{3} \text { where }\left[\mathbb{K}_{f_{6}}: \mathbb{Q}\right]=8,
\end{aligned}
$$




$$
\begin{aligned}
& \underset{\substack{q<500 \text { prime, } \\
q \equiv \pm 1 \text { mod } 31}}{\operatorname{gcd}}\left|A_{31, g_{1}}^{\prime}\left(\mathbb{F}_{q}\right)\right|=2^{2} \cdot 7 \text { where } \mathbb{K}_{g_{1}}=\mathbb{Q}(\sqrt{2}), \\
& \underset{\substack{q<500 \text { prime, } \\
q \equiv \pm 1 \text { mod } 31}}{\operatorname{gcd}}\left|A_{31, g_{2}}^{\prime}\left(\mathbb{F}_{q}\right)\right|=5 \cdot 11 \text { where } \mathbb{K}_{g_{2}}=\mathbb{Q}(\sqrt{5}), \\
& \underset{\substack{q<500 \text { prime, } \\
q \equiv \pm 1 \text { mod } 31}}{\text { gcd }}\left|A_{31, g_{3}}^{\prime}\left(\mathbb{F}_{q}\right)\right|=2302381 \text { where }\left[\mathbb{K}_{g_{3}}: \mathbb{Q}\right]=8, \\
& \underset{\substack{q<500 \text { prime, } \\
q \equiv \pm 1 \text { mod } 31}}{\operatorname{gcd}}\left|A_{31, g_{4}}^{\prime}\left(\mathbb{F}_{q}\right)\right|=31^{6} \text { where }\left[\mathbb{K}_{g_{4}}: \mathbb{Q}\right]=16 .
\end{aligned}
$$

So for $p=29$ and $p=31$ we have:

$$
\prod_{f} \underset{\substack{q<500 \text { prime } \\ q \equiv \pm 1 \bmod p}}{\operatorname{gcd}}\left|A_{p, f}^{\prime}\left(\mathbb{F}_{q}\right)\right|=\left|\mathfrak{C}_{n s}^{+}(p)\right|
$$

where the product runs over all equivalence classes of newforms.

\section{Acknowledgements}

I would like to express my gratitude to my advisor Prof. René Schoof for his valuable remarks during the development of this research work, especially for the last section.

\section{References}

[1] B. Baran, Normalizers of non-split Cartan subgroups, modular curves and the class number one problem, Journal of Number Theory, vol. 130, 2010, 2753-2772.

[2] Y. Bilu, P. Parent and M. Rebolledo, Rational points on $X_{0}\left(p^{r}\right)$, Ann. Inst. Fourier.

[3] I. Chen, The Jacobian of non-split Cartan modular curves, Proc. London Math. Soc. (3) 77 (1998), no.1, 1-38.

[4] F. Diamond and J. Shurman, A First Course in Modular Forms, Graduate Texts in Mathematics, vol.228, Springer Verlag, New York, 2005.

[5] V.G. Drinfeld, Two theorems on modular curves, Functional Analysis and its applications, Vol.7 No.2, translated from the Russian, AprilJune 1973, pp. 155-156. 
[6] H. Iwaniec, Topics in Classical Automorphic Forms, American Mathematical Society: Providence 1997.

[7] D. Kubert and S. Lang, Modular Units, Grundlehren der mathematischen Wissenschaften 244, Springer Verlag, New York-Berlin, 1981.

[8] D. Kubert and S. Lang, Units in the modular function field IV, The Siegel functions are generators, Math. Ann. 227 (1977) pp. 223-242.

[9] S. Lang, Elliptic functions, Addison Wesley, 1974.

[10] S. Lang, Introduction to Modular Forms, Springer Verlag, 1977.

[11] J. Manin, Parabolic points and zeta functions of modular curves, Izv.Akad.Nauk SSSR, Vol.6 No.1 (1972) American Mathematical Society translation pp. 19-64.

[12] B. Mazur, Rational isogenies of prime degree, Inv. Math. 44 (1978), 129-162.

[13] M. Rebolledo and C. Wuthrich, A moduli interpretation for the nonsplit modular curve, 2014.

[14] K.A. Ribet and W.A. Stein, Lectures on Modular Forms and Hecke Operators, 2011.

[15] J.P. Serre, Lectures on the Mordell-Weil Theorem, third ed., Aspects of Mathematics, Friedr. Vieweg \& Sohn, Braunschweig, 1997.

[16] J.P. Serre, Properiétés galoisienne des points d'ordre fini des courbes elliptiques, Invent. Math. 15, 259-331, 1972.

[17] G. Shimura, Introduction to the Arithmetic Theory of Automorphic Functions, Iwanami Shoten and Princeton University Press, 1971.

[18] C.L. Siegel, Lectures on advanced analytic number theory, Tata Institute Lecture Notes, 1961, 259-331.

[19] J. Silverman, Advanced topics in the arithmetic of elliptic curves, Graduate Texts in Mathematics 151 (1994), Springer

[20] L.C. Washington, Introduction to Cyclotomic Fields, Volume 83 of Graduate Texts in Mathematics, Springer-Verlag, 1982. 